\title{
Oncostatin M drives intestinal inflammation in mice and its abundance predicts response to tumor necrosis factor- neutralizing therapy in patients with inflammatory bowel disease
}

\author{
Nathaniel R. West ${ }^{1,2, \dagger}$, Ahmed N. Hegazy ${ }^{1,2, \dagger}$, Benjamin M. J. Owens ${ }^{3}$, Samuel J. Bullers ${ }^{1}$, \\ Bryan Linggi $^{4}$, Sofia Buonocore ${ }^{5}$, Margherita Coccia ${ }^{5}$, Dieter Görtz ${ }^{6}$, Sébastien This ${ }^{2}$, \\ Krista Stockenhuber ${ }^{1,2}$, Johanna Pott ${ }^{5}$, Matthias Friedrich ${ }^{1}$, Grigory Ryzhakov ${ }^{1}$, Frédéric \\ Baribaud $^{4}$, Carrie Brodmerkel ${ }^{4}$, Constanze Cieluch ${ }^{7}$, Nahid Rahman ${ }^{8}$, Gerhard Müller- \\ Newen $^{6}$, Raymond J. Owens ${ }^{8,9}$, Anja A. Kühl7 ${ }^{7}$, Kevin J. Maloy ${ }^{5}$, Scott E. Plevy ${ }^{4}$, Oxford IBD \\ Cohort Investigators ${ }^{10}$, Satish Keshav ${ }^{2}$, Simon P. L. Travis ${ }^{2}$, and Fiona Powrie ${ }^{1,2,{ }^{*}}$ \\ ${ }^{1}$ Kennedy Institute of Rheumatology, University of Oxford, Oxford, UK \\ ${ }^{2}$ Translational Gastroenterology Unit, Nuffield Department of Medicine, University of Oxford, \\ Oxford, UK \\ ${ }^{3}$ Somerville College, University of Oxford, Oxford, UK \\ ${ }^{4}$ Janssen Research and Development LLC, Raritan, NJ, USA
}

\footnotetext{
Users may view, print, copy, and download text and data-mine the content in such documents, for the purposes of academic research, subject always to the full Conditions of use:http://www.nature.com/authors/editorial_policies/license.html\#terms

*Address for correspondence Prof Fiona Powrie, Kennedy Institute of Rheumatology, University of Oxford, Roosevelt Drive, Headington, Oxford, OX3 7FY, UK. Fiona.powrie@kennedy.ox.ac.uk.

10 Individual investigators are listed at the end of the manuscript.

$\dagger_{\text {Co-first authors }}$
}

Data-availability statement

Publically available transcriptomic data used in this study are available at NCBI GEO: GSE57945, GSE59071, GSE4183, GSE38713, GSE16879, GSE12251, and GSE23597.

Competing Interests

NRW, ANH, BMJO, and FP are inventors of patents relating to OSM as a therapeutic target for IBD. NRW, ANH, BMJO, SPLT, and FP are shareholders in ImmElpis Pharma Ltd. SK has received honoraria and/or research support from Allergan, Abbvie, AstraZeneca, ChemoCentryx Inc., Dr Falk Pharma, Ferring, Gilead, GSK, Merck, Mitsubishi-Tanabe Pharma, Pfizer, and Vifor Pharma. SPLT has received research support from Abbvie, IOIBD, Lilly, UCB, Vifor, and the Norman Collison Foundation. SPLT receives consulting fees from Abbvie, Amgen, Biogen, Boehringer Ingelheim, Bristol-Myers Squibb, Celgene, Chemocentryx, Cosmo, Ferring, Giuliani SpA, GSK, Lilly, MSD, Neovacs, NovoNordisk, Norman Collison Foundation, Novartis, NPS Pharmaceuticals, Pfizer, Proximagen, Receptos, Shire, Sigmoid Pharma, Takeda, Topivert, UCB, VHsquared, and Vifor Pharma. SPLT has received speaker fees from Abbvie, Biogen, Ferring, and Takeda. BL, FB, CB, and SEP are employees of Janssen Research and Development LLC. SB and MC are currently employees of GSK. FP receives consultancy fees from Janssen and GSK (unrelated to this work).

Author contributions

NRW, ANH, BMJO, and FP contributed to study conception and design, data analysis, and manuscript production. NRW and ANH designed, executed, and analyzed experiments. SJB, CC, and AAK performed histological assays. BL, FB, CB, and SEP contributed clinical trial data and analysis. SB, MC, and KJM provided data on the cytokine requirements of the $H h+\alpha$ IL-10R model of IBD. DG and GM-N designed the OR-Fc protein construct. NR and RJO designed the strategy for OR-Fc production and generated the final protein. ST contributed to intestinal stromal cell experiments. KS and GR contributed to preclinical murine experiments. JP conducted mouse intestinal organoid experiments. MF contributed to establishment of human intestinal stromal cell cultures. SK and SPLT provided clinical guidance and contributed to patient sample collection. All authors read and approved the final manuscript.

Oxford IBD Cohort Investigators

Dr. Carolina Arancibia, Dr. Adam Bailey, Dr. Ellie Barnes, Dr. Beth Bird-Lieberman, Dr. Oliver Brain, Dr. Barbara Braden, Dr. Jane Collier, Dr. James East, Dr. Lucy Howarth, A/Prof. Satish Keshav, Prof. Paul Klenerman, A/Prof. Simon Leedham, Dr. Rebecca Palmer, Dr. Fiona Powrie, Dr. Astor Rodrigues, Prof. Alison Simmons, Dr. Peter Sullivan, Prof. Simon Travis, Prof. Holm Uhlig. 
${ }^{5}$ Sir William Dunn School of Pathology, University of Oxford, Oxford, UK

${ }^{6}$ Institute of Biochemistry and Molecular Biology, RWTH Aachen University, Aachen, Germany

${ }^{7}$ Medical Department, Division of Gastroenterology, Infectiology and Rheumatology, CharitéUniversitätsmedizin Berlin, Germany

${ }^{8}$ OPPF-UK, The Research Complex at Harwell, Rutherford Appleton Laboratory, Harwell, Oxford, UK

${ }^{9}$ Wellcome Trust Centre for Human Genetics, University of Oxford, Oxford, UK

\section{Abstract}

Inflammatory bowel diseases (IBD), including Crohn's disease (CD) and ulcerative colitis (UC), are complex chronic inflammatory conditions of the gastrointestinal tract that are driven by perturbed cytokine pathways. Anti-tumor necrosis factor-a (TNF) antibodies are a mainstay therapeutic approach for IBD. However, up to $40 \%$ of patients are non-responsive to anti-TNF agents, and identifying alternative therapeutic targets is a priority. Here we show that expression of the cytokine Oncostatin M (OSM) and its receptor (OSMR) is increased in the inflamed intestine of IBD patients compared to healthy controls, and correlates closely with histopathological disease severity. OSMR is expressed in non-hematopoietic, non-epithelial intestinal stromal cells, which respond to OSM by producing various pro-inflammatory molecules including interleukin-6 (IL-6), the leukocyte adhesion factor ICAM-1, and chemokines that attract neutrophils, monocytes, and T cells. In an animal model of anti-TNF resistant intestinal inflammation, genetic deletion or pharmacological blockade of OSM significantly attenuates colitis. Furthermore, high pretreatment OSM expression is strongly associated with failure of anti-TNF therapy based on analysis of over 200 IBD patients, including two cohorts from phase 3 clinical trials of infliximab and golimumab. OSM is thus a potential biomarker and therapeutic target for IBD, with particular relevance for anti-TNF resistant patients.

\section{Introduction}

IBD is an etiologically complex inflammatory disorder, involving the interaction of genetic predisposition, environmental triggers, microbial dysbiosis, and perturbation of immune homeostasis 1-4. Immune dysregulation in IBD depends on the inappropriate production of diverse pro-inflammatory cytokines, which orchestrate intestinal inflammation and constitute attractive targets for therapeutic development5,6. Indeed, blockade of TNF using monoclonal antibodies (anti-TNF therapy) is now firmly established as an effective therapeutic approach for IBD. Nevertheless, up to $40 \%$ of patients with IBD exhibit primary non-responsiveness to anti-TNF therapy, and many patients who are initially responsive develop therapeutic resistance 7,8 . Various other cytokines have been targeted in clinical trials (including interferon (IFN)- $\gamma$, IL-6, and IL-17A) but their blockade has generally resulted in negligible efficacy or, in the case of IL-17A, deleterious side effects in subgroups of patients5,9. Therefore, we sought to identify novel cytokines that could potentially serve as alternative therapeutic targets to TNF. In a large number of IBD patients, we identified OSM (Oncostatin M) as a highly expressed cytokine that is associated with anti-TNF resistant disease. Furthermore, OSM was found to promote intestinal pathology in an anti- 
TNF resistant mouse model of IBD. Intriguingly, OSM appears to promote intestinal inflammation by inducing chemokine, cytokine, and adhesion factor expression in gutresident stromal cells, which express high amounts of the OSM receptor- $\beta$ (OSMR).

OSM is part of the IL-6 cytokine family, which shares gp130 as a receptor subunit10. Depending on the cell type, human OSM can induce signalling via the JAK-STAT pathway (including JAK1, JAK2, STAT1, STAT3, STAT5, and possibly STAT6), the phosphatidylinositol-3-kinase (PI3K)-Akt pathway, and mitogen activated protein kinase (MAPK) cascades via heterodimeric receptors comprised of gp130 and either OSMR or leukemia inhibitory factor receptor- $\beta$ (LIFR) 11,12. By contrast, mouse OSM is thought to mediate similar signal transduction mainly via gp130-OSMR heterodimers11,12. OSM supports diverse homeostatic processes, including liver repair, cardiac tissue remodeling, osteoclastogenesis, and hematopoiesis11,12. However, overproduction of OSM is thought to promote a variety of pathologies, including skin and lung inflammation, atherosclerosis, and several forms of cancer11,12. Interestingly, a single-nucleotide polymorphism in the human OSM locus is strongly associated with risk of developing IBD13. Nevertheless, the role of OSM in IBD has remained unclear11,14,15.

\section{Results}

\section{OSM and OSMR are highly expressed in IBD}

To identify additional cytokines that may promote IBD pathogenesis, we analyzed cytokine mRNA expression in intestinal mucosal biopsies from previously published cohorts of patients with clinically active CD ( $n=162 \mathrm{CD}$ versus $n=42$ non-IBD controls; RNA-seq data) or UC ( $n=74 \mathrm{UC}$ versus $n=11$ non-IBD controls; Affymetrix microarray data)16,17. 64 candidate cytokines with data available in both studies were examined. Of these, only 4 were significantly enriched in inflamed tissue in both cohorts compared to non-IBD controls: IL 6 , $I L 1 A, I L 1 B$, and $O S M$ (Fig. 1a, Supplementary Table 1). Of these cytokines, OSM is the least well characterized in the gut, and we therefore chose to investigate it further. Among untreated paediatric patients with newly diagnosed CD16, we found $O S M$ to be the most highly and consistently expressed cytokine relative to healthy control mucosa (Fig. 1b, Supplementary Table 1). Furthermore, OSM was particularly enriched in patients with deep mucosal ulcerations (Fig. 1c). While OSMR was similarly enriched in IBD mucosa, this was not true of LIFR or IL6ST (gp130) (Fig. 1c).

To validate our initial findings, we used quantitative real-time PCR (Q-PCR) to examine $O S M$ and $O S M R$ expression in freshly isolated biopsies from IBD patients and healthy controls who underwent routine endoscopy at the John Radcliffe Hospital, Oxford (for associated clinical information, see Supplementary Table 2). This confirmed high expression of $O S M$ and $O S M R$ in tissue from IBD patients with active disease (Fig. 1d), and also revealed a close correlation between OSMIOSMR expression and histopathological disease severity (Fig. 1e). No difference in expression of either OSM or OSMR was observed in patients with CD or UC (Fig. 1f). Analysis of transcriptomic data from four different countries 16-20 further confirmed that $O S M$ and $O S M R$ are consistently over-expressed in the intestinal mucosa of patients with active IBD (total control $n=99$, total IBD $n=370$; Supplementary Table 3). Neither OSM nor OSMR expression correlated with standard 
clinical parameters including gender, age at diagnosis, disease duration, serum c-reactive protein (CRP), peripheral blood leukocyte count, or treatment with pharmacological therapies; however, OSM and OSMR expression was increased in patients with IBD who required surgery, suggesting an association with treatment-resistant or complicated disease (Supplementary Fig. 1).

\section{OSM expression is associated with response to TNF-neutralizing therapy}

Hierarchical clustering of cytokine and chemokine expression in two IBD cohorts revealed that OSM is consistently associated with a discrete module of inflammatory mediators (Fig. 2a, Supplementary Fig. 2). Although molecular correlates of anti-TNF response have been reported previously, there are currently no clinically accepted predictive biomarkers of antiTNF response for IBD18,21-23. We therefore asked whether the OSM-associated inflammatory module was associated with responsiveness to anti-TNF therapy. Among patients refractory to corticosteroids or other immunosuppressive therapies, unsupervised hierarchical clustering based on expression of OSM-associated module genes revealed that high module expression in pre-treatment biopsies was strongly associated with primary nonresponsiveness to anti-TNF therapy (Fig. 2b, Supplementary Fig. 3a-b). Complete mucosal healing (based on endoscopic and histological criteria) following infliximab (Remicade) therapy was achieved by $69-85 \%$ of patients with low OSM module expression, but was observed in only 10-15\% of those with high OSM module expression (Fig. 2c,

Supplementary Fig. 3b). Notably, baseline expression of OSM and OSMR alone was strongly associated with poor primary response to infliximab in three different cohorts (Fig. 1d, Supplementary Fig. 3c-d)18,22,23. Indeed, at the whole transcriptome level, OSM was among the 20 most strongly expressed genes in anti-TNF resistant patients compared to antiTNF responders (Supplementary Table 4). Baseline OSM and OSMR expression was also elevated in patients who responded initially to infliximab but relapsed by week 30 posttreatment (Supplementary Fig. 3d).

The association between mucosal $O S M$ expression and anti-TNF response was confirmed in two additional prospective patient cohorts from phase 3 clinical trials of moderate-toseverely active UC, one treated with intravenous infliximab (Fig. 2e-g), and the other treated with subcutaneous golimumab (Simponi), an alternative anti-TNF agent (Supplementary Fig. 3e-i)24,25. Unlike the cohorts described above, these trials categorized patients into response groups on the basis of improvement in clinical Mayo scores. This allowed us to assess patients who achieved full remission following therapy, those who partially responded (an improvement in Mayo score, but with remaining disease activity), and those who were completely non-responsive to therapy. While OSM was clearly most highly expressed in the non-responsive group of infliximab-treated patients (Fig. 2f), it was significantly elevated in both non-responders and partial responders who received golimumab (Supplementary Fig. 3f). Although OSM correlates broadly with inflammation severity in the overall IBD population (Fig. 1e), OSM did not correlate substantially with disease severity in these clinical trial cohorts, which were comprised exclusively of patients with high disease activity (Fig. 2g, Supplementary Fig. 3h). In addition, baseline disease activity and clinical biomarker expression in these trials was not significantly associated with treatment response (Fig. 2e, Supplementary Fig. 3e, g), suggesting that OSM measurement prior to therapy 
could yield useful prognostic information that cannot be obtained from conventional clinical assessment. Thus, analysis of five datasets (comprising 227 patients) demonstrates that high baseline $O S M$ expression in the intestinal mucosa is reproducibly associated with decreased responsiveness to anti-TNF therapy.

\section{Human intestinal stromal cells express high amounts of OSMR}

To gain insight into the role of OSM in the intestine, we conducted gene ontology network analysis of human IBD transcriptomic data using ClueGo26. Relative to TNF-high tissues (used as a control for generic inflammation), OSM-high and OSMR-high tissues were strongly enriched in genes related to leukocyte chemotaxis, extracellular matrix organization, and mesenchymal development, suggesting that OSM may influence nonhematopoietic stromal cells (Supplementary Fig. 4). Indeed, in mucosal biopsies from healthy donors and IBD patients analyzed using Q-PCR, OSMR expression correlated closely with the well-established fibroblast products COL1A1 (collagen 1A1) and FAP (fibroblast activation protein-a), as well as the lymphoid tissue-like stromal markers $P D P N$ (podoplanin/gp38) and ICAM1 (intercellular adhesion molecule-1; Fig. 3a) 27-32. All of these stromal genes were highly expressed in inflamed IBD specimens (Fig. 3b).

Furthermore, stromal cells with high PDPN expression were abundant in the colon lamina propria of patients with CD or UC (Fig. 3c).

Flow cytometry analysis of human intestinal mucosa revealed that OSMR is undetectable in epithelial and hematopoietic cells, expressed in low amounts by endothelial cells, and strongly expressed by the majority of $\mathrm{CD}^{-} 5^{-} \mathrm{EpCAM}^{-} \mathrm{CD} 31^{-}$stromal cells (Fig. 3d-f). In contrast, OSM was expressed by various hematopoietic populations in human intestinal mucosa, including $\mathrm{CD}^{+}{ }^{+} \mathrm{T}$ cells and HLA-DR ${ }^{+}$antigen presenting cells from both non-IBD controls and IBD patients (Supplementary Fig. 5a-d). Notably, OSMR was expressed more abundantly than the related IL-6 receptor by intestinal stromal cells from both non-IBD control and IBD patients (Supplementary Fig. 5g). Consistent with the high amounts of OSMR produced by stromal cells, OSM stimulation elicited phosphorylation of STAT3, STAT1, Akt, and ERK1/2 MAP kinases in CCD18Co cells (primary human intestinal stroma), whereas IL-6 triggered only STAT3 phosphorylation (Fig. 3g). OSMR expression in different cell populations was equivalent in healthy control and IBD patients in terms of both the frequency of OSMR ${ }^{\text {high }}$ cells (Fig. 3h) and OSMR expression intensity (Fig. 3i). This suggests that the increased OSMR expression in biopsies from IBD patients (Fig. 1) is due to an accumulation of OSMR-expressing stromal cells in the tissue, not increased OSMR expression per cell. Because stromal cells vastly outnumber endothelial cells in the intestine (Supplementary Fig. 5h) and are highly enriched in OSMR, stromal cells appear to be the dominant intestinal OSMR ${ }^{+}$population. Intriguingly, OSMRhigh stromal cells co-expressed PDPN and ICAM-1, a phenotype that is similar to fibroblastic reticular cells (FRCs) in secondary lymphoid tissue (Fig. 3j) 33 .

\section{OSM promotes inflammatory activity in the intestinal stroma}

To assess the response of intestinal stromal cells to OSM, we treated CCD18Co cells with recombinant human OSM and used Q-PCR to profile expression of the OSM-associated inflammatory module (see Fig. 2a). Notably, half of the module members were directly 
induced by OSM stimulation, including IL 6 and a functionally diverse set of chemokines (Fig. 4a). OSM also induced expression of ICAM-1 and PDPN, suggesting that it may enforce the phenotype of OSMR ${ }^{\text {high }}$ stroma observed in vivo (Fig. 3j). The OSM response was strictly dependent on OSMR, but not LIFR (Supplementary Fig. 5i-j).

To determine if a similar OSM response can be detected in vivo, we used fluorescence activated cell sorting (FACS) to purify OSMR ${ }^{\text {high }}$ and OSMR ${ }^{\text {low }}$ stromal cells from colon tissue of 3 donors (Fig. 4b, Supplementary Fig. 5k). Q-PCR analysis of the purified cell populations revealed similar expression of classical fibroblast products including collagens, lumican, fibronectin, and CD90 (Fig. 4c, Supplementary Fig. 51). However, relative to OSMR $^{\text {low }}$ cells, OSMR ${ }^{\text {high }}$ stroma expressed high amounts of several chemokines and cytokines including $I L 6, C C L 2, C X C L 1$, and $C X C L 10$, consistent with the gene induction profile observed in CCD18Co cells (Fig. 4c). This suggests that high OSMR expression identifies a more immunostimulatory subset of intestinal stromal cells.

Because OSM is known to synergize with other inflammatory cytokines (in breast cancer, for example), we asked whether combined OSM and TNF stimulation have synergistic effects in human intestinal stroma. Indeed, some genes (such as the CXCR3 ligands CXCL9/10/11) were synergistically induced in CCD18Co cells by combined OSM and TNF treatment, while others such as CCL2 were not (Fig. 4d, Supplementary Fig. 5m). Comparable responses were observed in primary ex vivo cultures of human colonic stromal cells (Fig. 4e, Supplementary Fig. 5n). Compared to stromal cells, HUVEC (human umbilical vein endothelial cells) were weakly responsive to OSM, consistent with lower endothelial expression of OSMR (Supplementary Fig. 5o-p). Intriguingly, stronger responses to OSM and TNF treatment were observed in primary stromal cultures from IBD patients compared to cultures from non-IBD controls (Fig. 4f).

\section{The OSM-stromal cell axis is conserved in mice}

To explore the OSM-stromal axis in a relevant pre-clinical setting, we used a model IBD system driven by oral Helicobacter hepaticus infection and systemic IL-10 receptor blockade in wild type C57BL/6 mice (Hh+aIL-10R model, Fig. 5a)34,35. This causes T celldependent pathology that is resistant to anti-TNF therapy (Supplementary Fig. 6a)36. Furthermore, this model does not require signaling by IL-6, IL-1 $\alpha$, or IL-1 $\beta$ (Supplementary Fig. 6b-c). At peak disease severity, colon lamina propria leukocytes increased 10-fold in abundance (Fig. 5b). Osm and Osmr were highly expressed in the colons of colitic mice (Fig. 5c), and OSM protein was readily detectable in colon explant supernatants and fecal matter of inflamed animals (Fig. 5d). The in vivo expression kinetics of OSM closely mirrored those of IL-6, IL-1 $\beta$, and TNF, but differed substantially from IL-23, which is required at early time-points for induction of colitogenic $\mathrm{T}$ cell responses (Supplementary Fig. 6d)34,37.

Like humans, Osm displayed a hematopoietic expression pattern with relative enrichment in antigen presenting cells, based on Q-PCR analysis of FACS-sorted colon populations from healthy and colitic mice (Fig. 5e, Supplementary Fig. 6e). In contrast, Osmr expression was restricted to intestinal stromal cells in both healthy and inflamed animals (Fig. 5e). The colon stroma from inflamed mice also expressed high amounts of $I I 1 \mathrm{~b}$ and $I I 6$, suggesting 
that these cells adopt a pro-inflammatory state during colitis (Fig. 5e). Furthermore, the colon lamina propria of colitic mice was highly enriched in $\mathrm{PDPN}^{+}$stromal cells, consistent with human IBD (Fig. 5f). To determine the location of OSMR-expressing cells in the intestine, we used RNAscope in situ hybridization. Osmr expression in healthy mouse colon tissue was detected in endothelial and stromal cells, the latter distributed widely within the lamina propria along the entire length of the crypt-villus axis, as well as within lymphoid clusters. No expression was observed in epithelial cells, and a similar expression pattern was observed in the ileum (Supplementary Fig. 7a). Consistent with the increased numbers of $\mathrm{PDPN}^{+}$stromal cells and increased OSMR expression in inflamed colon tissue, the number of cells expressing Osmr was markedly increased in the lamina propria of colitic mice (Fig. $5 \mathrm{~g}$ ). Mouse colon stromal cells responded strongly to OSM in a manner similar to that of human stroma (Supplementary Fig. 7). In contrast, substantial OSM responsiveness was not observed in $\mathrm{CD} 45^{+}$leukocytes from mouse spleen or colon, or from mouse colonic epithelial organoids (Supplementary Fig. 7). Taken together, these data indicate that intestinal OSM biology of healthy and colitic mice is largely consistent with that seen in humans.

\section{OSM drives colitis in a pre-clinical model of anti-TNF resistant IBD}

To determine if OSM can influence anti-TNF resistant colitis, we compared OSM-deficient mice $\left(\mathrm{Osm}^{-1-}\right)$ to co-housed wild type littermates using the $\mathrm{Hh}+\mathrm{aIL}-10 \mathrm{R}$ model. At steady state, $\mathrm{Osm}^{-1-}$ mice showed normal organ histology (Supplementary Fig. 8a), normal development of secondary and mucosal-associated lymphoid tissue (Supplementary Fig. 8bd), a normal leukocyte repertoire in lymphoid and intestinal tissue, and normal frequencies of non-hematopoietic cells in the colon (Supplementary Fig. 9). At peak disease severity, $\mathrm{Osm}^{-1-}$ mice displayed reduced colon pathology based on colonoscopy and histological assessment compared to wild type controls, particularly with regard to severe disease features such as crypt abscess formation, submucosal inflammation, and edema (Fig. 6a-d). This was not due to differences in H. hepaticus colonization (Supplementary Fig. 9k).

Notably, $\mathrm{Osm}^{-1-}$ colons displayed normal activation of chemokine and cytokine expression during the first week of colitis, as well as normal accumulation of leukocyte populations in the lamina propria (Fig. 6e, Supplementary Fig. 10a-d). However, this response was attenuated during week 2 , in parallel with reduced accumulation of $\mathrm{CD}^{+} \mathrm{T}$ cells and granulocytes. Reduced proliferation of colonic $\mathrm{CD}^{+}{ }^{+} \mathrm{T}$ cells (determined by Ki-67 staining) could not explain the differences in T cell abundance, and leukocyte accumulation in $\mathrm{Osm}^{-1-}$ mesenteric lymph nodes was entirely normal (Supplementary Fig. 10e-f). This suggests that OSM has little influence on the early/acute phase of inflammation, but enhances inflammation at later time points by promoting stromal chemokine production and selective recruitment of $\mathrm{CD}^{+} \mathrm{T}$ cells and granulocytes. Indeed, to confirm that trafficking of $\mathrm{Osm}^{-1-}$ leukocytes during acute inflammation is normal, we employed a model of skin inflammation that involves topical application of imiquimod (a toll-like receptor 7 agonist) to mouse ears over 6 days. Consistent with acute colon inflammation, $\mathrm{Osm}^{-1-}$ and wild type littermates showed equivalent skin thickening and recruitment of monocytes, granulocytes, and $\mathrm{T}$ cells to skin and cervical lymph nodes (Supplementary Fig. 10g-1). Notably, in the Hh+aIL-10R colitis model, $\mathrm{Osm}^{-1-}$ mice displayed reduced colon expression of the OSM-associated 
inflammatory module that is associated with anti-TNF resistance in humans (Supplementary Fig. 11a).

\section{OSM neutralization suppresses anti-TNF resistant colitis in mice}

To test the therapeutic utility of OSM, we treated wild type C57BL/6 mice with an Fctagged soluble OSMR-gp130 fusion protein (OR-Fc; Fig. 6f, Supplementary Fig. 11b)38 starting at day 7 of the $H h+a$ IL-10R protocol, by which time colitis is readily detectable (Supplementary Fig. 11c). Compared to commercially available polyclonal anti-OSM antibodies, the OR-Fc construct was more efficient at neutralizing OSM in an ex vivo mouse intestinal stromal cell assay (Supplementary Fig. 11b). OR-Fc treatment significantly reduced colitis severity compared to mock treatment (recombinant $\mathrm{Fc}$ protein), demonstrating the potential utility of OSM as a therapeutic target (Fig. 6g-h, Supplementary Fig. 11d). Consistent with $\mathrm{Osm}^{-1-}$ mice, colonic expression of the clinically relevant OSMassociated inflammatory module during colitis was suppressed by therapeutic OSM blockade (Fig. 6i).

\section{Discussion}

IBD is a clinically challenging illness that strikes at a young age and causes life-long morbidity. The high rate of primary and acquired resistance to therapy makes IBD a significant area of unmet medical need, for which alternative therapeutic options and improved patient stratification methods are required. Although cytokines are well known to mediate intestinal inflammation, few have proven useful as therapeutic targets5. The notable exception is TNF, neutralization of which has been profoundly successful for treating IBD. More recently, the IL-12/IL-23 neutralizing antibody ustekinumab has also shown clinical efficacy for CD39. Nevertheless, treatment resistance remains a significant clinical challenge, making the identification of alternative therapeutic targets an urgent priority.

To identify such targets, we profiled cytokine expression in healthy individuals and patients with IBD. This revealed OSM to be consistently overexpressed in inflamed intestinal tissue from mice and humans. Hematopoietically derived OSM appears to mediate intestinal pathology by promoting inflammatory behavior in gut-resident stromal cells, revealing a novel system of leukocyte-stromal cell crosstalk that may have relevance in multiple mucosal tissues. OSM is expressed as part of a core inflammatory cytokine module including IL- 6 and IL- $1 \alpha / \beta$, with effects that are distinct from the closely related IL- 6 and synergistic with those of TNF. Whereas IL-23 is a critical trigger of bacterially driven colitis through its actions on T cells34,37, OSM may act as an inflammatory amplifier and driver of disease chronicity by promoting chemokine, cytokine, and adhesion factor production by intestinal stromal cells (Supplementary Fig. 11e). Whether OSM can influence tissue fibrosis via the stromal compartment remains to be determined. Intriguingly, OSM has been shown to bind extracellular matrix components (including collagen, laminin, and fibronectin) in a manner that protects it from proteolytic degradation and maintains biological activity for prolonged periods of time40. This system could amplify the effects of OSM in chronic inflammation by promoting accumulation of stable OSM protein, particularly in tissues with high amounts of extracellular matrix deposition. 
The immunological importance of intestinal stromal cells is not well understood; while they can respond to microbial challenges and influence dendritic cell function under steady state conditions27,29, a critical inflammatory role for stromal cells in IBD has not been demonstrated28. Nevertheless, data from other inflammatory diseases such as rheumatoid arthritis support the hypothesis that stromal cells are active contributors to immune pathology 41. The high frequency of fibrotic complications in CD is consistent with a pathological role for intestinal stromal cells. However, we found that inflamed tissue from both CD and UC patients contains large numbers of PDPN ${ }^{+}$stromal cells, despite UC not being strongly associated with fibrosis. Similarly, mice subjected to $H h+\mathrm{aIL}-10 \mathrm{R}$ colitis show a substantial expansion of the intestinal stromal cell compartment in the absence of overt intestinal fibrosis. These observations highlight the possibility that intestinal stromal cells may contribute to inflammatory pathology in ways that extend beyond the simple deposition of extracellular matrix components. A major unanswered question regarding intestinal stromal cells is their ontogeny. Although the number of $\mathrm{PDPN}^{+}$stromal cells clearly increases during intestinal inflammation in both mice and humans, it is unknown whether they arise from expansion of tissue-resident precursors, are recruited from elsewhere (e.g. via circulating precursors), or if they differentiate from a distinct tissueresident cell type. Similarly, it is not known if OSMR ${ }^{\text {high }}$ intestinal stromal cells represent a distinct mesenchymal lineage, or simply a state of increased immunological activity. Also noteworthy is the heightened cytokine sensitivity of intestinal stromal cells from IBD patients. Although the mechanism underlying this difference is not clear, it may be related to a similar phenomenon observed in fibroblast-like synoviocytes from rheumatoid arthritis patients, which display imprinted hyperresponsiveness to inflammatory stimuli, possibly due to mutations and/or epigenetic alterations 41

To assess the role of OSM in a preclinical model of IBD, we chose to employ the $H h$ $+a$ IL-10R system. Although several murine models of IBD exist, we chose this system for the following reasons: (a) it does not require the use of genetically modified mice, which precludes the possibility of confounding developmental defects; (b) similar to current concepts of human IBD etiology, it requires dual triggers in the form of infection with the commensal pathobiont $H$. hepaticus and transient immune dysregulation via IL-10R blockade; (c) it is driven by a full spectrum of innate and adaptive immune processes, as occurs in human IBD patients; and (c) it is highly resistant to TNF blockade, making it ideal for investigating novel drivers of colitis. We have also observed high expression of OSM in additional mouse models of IBD, including chemically induced colitis and adoptive transfer of naïve CD4+ ${ }^{+}$cells to $\mathrm{Rag}^{-/-}$hosts, suggesting that OSM may be relevant beyond the $H h$ + aIL-10R model.

In addition to alternative therapeutic targets, IBD patients would benefit substantially from improved systems for predicting disease course and response to therapy. In the case of antiTNF therapy, no biomarkers are currently used for predictive purposes in standard clinical practice, and conventional clinical parameters are insufficient to predict therapeutic response21. This forces caregivers to make treatment decisions with little knowledge of whether a patient is likely to benefit, placing many patients at unnecessary risk of developing anti-TNF related complications (e.g. infections) 42,43 , and inflating the economic burden of IBD care. However, we have observed that high OSM expression in 
intestinal mucosa is reproducibly associated with a high risk of resistance to anti-TNF therapy. Our data thus highlight the potential for developing a robust assay-based on measuring expression of OSM or similar inflammatory factors-that could assist clinicians in determining whether to prescribe anti-TNF antibodies or explore alternative therapeutic options.

While OSM can influence tissue remodeling in organs such as the heart and liver44-48, $\mathrm{Osm}^{-1-}$ mice are viable and healthy, suggesting that therapeutic blockade of OSM may cause minimal side effects. Indeed, OSM has been targeted for rheumatoid arthritis in phase I and II clinical trials using a humanized anti-OSM monoclonal antibody (GSK315234)49. Although little clinical efficacy was observed, the drug was well tolerated, with a doserelated decrease in platelet counts being the most notable adverse effect (although all platelet counts remained within the normal reference range). This is consistent with a prior report that OSMR-deficient mice have modestly reduced platelet counts due to a reduction in bone marrow megakaryocyte progenitors50. Because $\mathrm{Osm}^{-1-}$ mice have reduced nociception51 and OSM has been implicated in other inflammatory disorders such as psoriasis52-55 and arthritis56-59, which are common comorbidities of IBD60, it is tempting to speculate that OSM blockade could also be beneficial in managing extra-intestinal manifestations of IBD. OSM and OSMR are over-expressed in the vast majority of active IBD lesions, particularly in patients with anti-TNF resistant disease. OSM could therefore be a novel predictive biomarker and therapeutic target for this clinically challenging population, and clinical studies to evaluate this hypothesis are warranted.

\section{Methods}

\section{Human samples and cell isolation}

Intestinal pinch biopsies and surgical resection specimens were obtained from healthy donors or IBD patients attending the John Radcliffe Hospital Gastroenterology Unit (Oxford, UK). Biopsies were collected during routine endoscopy; resections were obtained from patients with IBD undergoing surgery for severe disease, chronically active disease, or complications of disease. Some non-IBD (non-inflamed) control specimens were obtained from normal regions of bowel adjacent to resected colorectal tumors. Informed, written consent was obtained from all donors. Human experimental protocols were approved by the NHS Research Ethics System (Reference numbers: 09/H0606/5 for IBD patients and 11/YH/ 0020 for controls). Tissues were prepared as previously described with minor modifications61. In brief, mucosa was dissected and washed in 1mM DTT (dithiothreitol) solution for 15 minutes at room temperature to remove mucus. Specimens were then washed three times in $0.75 \mathrm{mM}$ EDTA (ethylenediaminetetraacetic acid) to deplete epithelial crypts and digested overnight in $0.1 \mathrm{mg} / \mathrm{ml}$ collagenase A solution (Roche, UK). In some experiments, tissues were rapidly digested for 2 hours using $1 \mathrm{mg} / \mathrm{ml}$ collagenase A. For enrichment of mononuclear cells, digests were centrifuged for 30 minutes in a four-layer Percoll gradient and collected at the $40 \% / 60 \%$ interface. Stromal cells were collected at the $30 \% / 40 \%$ interface or were analyzed in unfractionated samples. Stromal cells were cultured ex vivo as described27. All solutions used were supplemented with antibiotics $(10,000 \mathrm{U} / \mathrm{ml}$ 
penicillin/streptomycin, $40 \mu \mathrm{g} / \mathrm{ml}$ gentamicin, $10 \mu \mathrm{g} / \mathrm{ml}$ ciprofloxacin, and $0.025 \mu \mathrm{g} / \mathrm{ml}$ amphotericin B (Sigma Aldrich, UK)).

\section{Human mucosal inflammation scoring}

Inflammation severity of human intestinal mucosa was classified by both endoscopic and histological criteria. Endoscopic classification was binarized into either inflamed or uninflamed categories based on assessment by the endoscopist. Where possible, matched biopsies were collected from both active lesions and macroscopically normal tissue at a distance from lesions. Endoscopic assessment was complemented by routine histopathological scoring by a gastrointestinal pathologist. Tissues were classified as quiescent (normal appearance), mildly inflamed, or severely inflamed.

\section{Analysis of transcriptomic data}

Whole transcriptome data were downloaded from the Gene Expression Omnibus website (http://www.ncbi.nlm.nih.gov/geo/). Before analysis, data for genes of interest were mediannormalized and $\log _{2}$ transformed. When genes in microarray data were represented by multiple probes, the probe with the greatest interquartile range was selected for analysis. The following publically available datasets were used in this study: GSE57945 (ileal biopsies from pediatric healthy controls and patients with newly diagnosed ileal $\mathrm{CD}$, colonic $\mathrm{CD}$, and UC)16, GSE59071 (UC)17, GSE4183 (undefined IBD)19, GSE38713 (UC)20, GSE16879 (matched pre-therapeutic and post-therapeutic biopsies of infliximab treated UC and CD)18, GSE12251 (UC before infliximab therapy)22, and GSE23597 (UC before and after infliximab therapy)23. Unsupervised hierarchical clustering was performed using Gene Cluster 3.0, with complete linkage as the clustering method and Euclidean distance as the similarity metric.

In addition to the publically available datasets described above, we analyzed OSM expression using unpublished transcriptomic data from two groups of patients with moderate-to-severe UC who were part of anti-TNF clinical trials (ClinicalTrials.gov Identifiers NCT00207688 and NCT00487539). Patients from NCT00207688 were part of the ACT1/2 clinical trials of intravenous infliximab therapy24, and samples were collected and processed as described by Toedter et al (2011)23. Patients from NCT00487539 were part of the PURSUIT trial of subcutaneous golimumab therapy25. Briefly, mucosal colonic biopsies were collected at weeks 0 and 6 during endoscopy from a sub-group of PURSUIT patients at 15 to $20 \mathrm{~cm}$ from the anal verge. Colon biopsies were also obtained from normal subjects who did not participate in the PURSUIT study to serve as controls. Informed consent was obtained from healthy individuals to undergo additional colonic biopsies for research purposes during colonoscopic procedures performed as part of routine clinical care (such as colorectal neoplasia screening or evaluation of gastrointestinal symptoms). The procedure verified that these individuals did not have inflammatory conditions of the gastrointestinal tract including IBD.. The normal colon samples were obtained from University of Pennsylvania School of Medicine (Philadelphia, PA) and University Hospital Gasthuisberg (Leuven, Belgium). Normal colon sample collection complied with the Principles of the Declaration of Helsinki and had ethics approval by the respective institutional review boards. Following collection, the patient and normal samples were 
preserved in RNAlater (Applied Biosystems, Foster City, CA). All biopsies were stored at $80{ }^{\circ} \mathrm{C}$ until RNA isolation was performed, which may have been up to 2 years following collection. RNA was isolated and hybridized to the GeneChip HT HG-U133+ PM Array (Affymetrix, Santa Clara, CA). Expression intensities were obtained from the Robust Multiarray Average (RMA) algorithm. The microarray data were pre-processed and normalized by Robust Multi-array Average using Array Studio software version 4.2 (OmicSoft Corp., St. Morrisville, NC). For analysis of OSM expression, Affymetrix probeset 230170_PM_at was used.

\section{Definition of response to anti-TNF therapy}

The criteria for determining primary responsiveness to anti-TNF therapy are described in the original reports of the GSE12251, GSE16879, and GSE23597 cohorts18,22,23. Briefly, patients with active IBD refractory to corticosteroids and/or immunosuppression underwent colonoscopy (with biopsy collection) within a week prior to anti-TNF therapy. For cohorts GSE12251 and GSE16879, response following treatment was defined as complete mucosal healing by both endoscopic and histological criteria. For GSE23597, treatment response was defined as a reduction from the baseline Mayo score of at least 3 points and at least 30\%, with reduction in the rectal bleeding subscore of at least 1 point or an absolute rectal bleeding score of 0 or 1 . For the ACT1/2 (NCT00207688)24 and PURSUIT (NCT00487539)25 clinical trials, response categories were defined as follows: clinical remission (post-treatment Mayo score of 0-2); partial clinical response (decrease from baseline Mayo score by $\geq 30 \%$ and $\geq 3$ points, but post-treatment score $\geq 3$ ); or no clinical response (decrease from baseline Mayo score of $<30 \%$ and/or $<3$ points). It should be noted that the definition of responsiveness in GSE23597 and the clinical trials was less robust than in the GSE12251 and GSE16879 discovery cohorts, where remission was defined strictly by the presence or absence of histologically evident inflammation following therapy.

Mice

Wild type C57BL/6, C57BL/6. $\mathrm{Osm}^{-1-}$, and C57BL/6.I11r $\mathrm{r}^{-1-}$ mice were bred and maintained under specific pathogen free conditions in accredited animal facilities at the University of Oxford. C57BL/6. $\mathrm{Osm}^{-1-}$ mice were acquired from the Jackson Laboratory (Maine, USA, stock \# 022338) and C57BL/6.II1r $1^{-1-}$ mice were a kind gift of Dr. Vincenzo Cerundolo. All procedures were conducted in accordance with the UK Scientific Procedures Act of 1986. Mice were negative for Helicobacter species and other known intestinal pathogens, were age and sex-matched, and more than 6 weeks old when first used. Both male and female mice were used in approximately equal proportions for all experiments. Mice were randomized to different treatments and all treatments were represented in a given cage of animals. In experiments involving $\mathrm{Osm}^{-/-}$mice, knockout animals and wild type littermate controls were co-housed. Experiments were replicated in two independent animal facilities within Oxford to control for differences in housing conditions. Minimum sample sizes for individual experiments were determined based not on a statistical method, but on experience with colitis models: $n=3$ for steady state animals and $n=6$ for animals given experimental colitis. 


\section{$H h+a l L-10 R$ colitis and in vivo treatments}

Experimental colitis was induced as described 34,35 . Briefly, mice were fed $1 \times 10^{8}$ colony forming units (c.f.u.) of $H$. hepaticus by oral gavage delivered with a $22 \mathrm{G}$ curved blunted needle on days 0 and 1 of the experiment. $1 \mathrm{mg}$ of an IL-10R blocking antibody (clone 1B1.2) was administered as an intraperitoneal injection once weekly starting at day 0 . In this model, disease severity peaks after 14 to 21 days and slowly resolves thereafter. To neutralize OSM in vivo, mice were treated with a previously described OSM receptor fusion protein $(\mathrm{OR}-\mathrm{Fc}) 38$. For increased in vivo stability, this construct was tagged with the $\mathrm{Fc}$ region of mouse IgG2A. OR-Fc was administered as a $150 \mu \mathrm{g}$ intraperitoneal injection every 2 days (equivalent to approximately $6 \mathrm{mg} / \mathrm{kg}$ ). Molar-equivalent doses of IgG2A-Fc (manufactured under the same conditions as $\mathrm{OR}-\mathrm{Fc}$ ) were used as control treatments. Some mice were also treated with a TNF-neutralizing antibody (clone XT3.11, Bio X Cell, USA) at a total weekly intraperitoneal dose of $1 \mathrm{mg}$ per animal. This dose was found to completely abrogate intestinal pathology in 129SvEv. $\mathrm{Rag}^{-1-}$ mice infected with H. hepaticus (not shown). For experiments involving anti-IL6R treatment and C57BL/6.IIIr $1^{-1-}$ mice, antiIL10R was injected once per week (starting at day 0 ) and animals were sacrificed after 4 weeks. Similarly, anti-IL6R (clone D7715A7) was administered once per week as a $1 \mathrm{mg}$ intraperitoneal dose starting at day 0 .

\section{Scoring of mouse colitis}

Colonoscopy to assess colitis severity was performed and scored according to the methods of Becker et al62. Histological assessment of colitis severity was performed as described63. Briefly, formalin-fixed paraffin-embedded cross-sections of proximal, middle, and distal colon were stained with hematoxylin and eosin and graded on a scale of 0 to 3 for four parameters: epithelial hyperplasia and goblet cell depletion, leukocyte infiltration, area affected, and features of severe disease activity. Common severity features include crypt abscess formation, submucosal leukocyte infiltration, and interstitial edema. Scores for each criterion are added to give an overall score of 0 to 12 per colon section. Data from the three colon regions are then averaged to give an overall score. Scoring was conducted in a blinded fashion and confirmed by an independent blinded observer. Interobserver Pearson correlation coefficients ranged from 0.90 to 0.95 .

\section{Mouse colon tissue preparation and cell isolation}

Mouse colons were washed with EDTA to remove epithelium and digested with collagenase VIII to liberate cell populations as described64. Tissue digests were separated by centrifugation on a $30 \% / 40 \% / 70 \%$ percoll gradient. Cells at the $30 \% / 40 \%$ interface were collected as the stroma/epithelium-enriched fraction, whilst cells at the $40 \% / 70 \%$ interface were collected as the lamina propria leukocyte enriched fraction. For ex vivo stromal culture, stromal fractions were plated and cultured as described35.

\section{Colon explant cultures}

Mouse proximal colon segments $\left(0.25 \mathrm{~cm}^{2}\right)$ were cultured overnight in RPMI media with $10 \%$ fetal calf serum (FCS) and 10,000 U/ml penicillin/streptomycin. OSM was quantified 
in the supernatant by enzyme-linked immunosorbent assay (ELISA, R\&D Systems, UK) and normalized to explant weight.

\section{Stimulation of stromal and endothelial cells}

CCD18Co cells (primary human intestinal fibroblasts; ATCC, not currently listed as misidentified on the ICLAC database) and primary ex vivo stromal cultures were grown in humidified incubators with $5 \% \mathrm{CO}_{2}$ at $37^{\circ} \mathrm{C}$ in DMEM media (Sigma) with $10 \%$ FCS and $2 \%$ human serum (Sigma). HUVEC cells (Gibco) were cultured as above in Medium 200 with low serum growth supplement (Gibco) according to manufacturer instructions. For cytokine stimulation experiments, unless otherwise indicated, all treatments were for two hours, and all cytokines were administered at a concentration of $10 \mathrm{ng} / \mathrm{ml}$. Cells were cultured for no more than 8 passages to avoid onset of senescence, phenotypic drift, and contamination (e.g. by mycoplasma). All cytokines were purchased from Peprotech. To knock down OSMR expression in CCD18Co cells, Accel SMARTpool siRNA was used following manufacturer instructions (GE Healthcare Dharmacon, USA), and cells were stimulated to test for OSM sensitivity after 72 hours of transfection.

\section{RNA extraction, cDNA synthesis, and qPCR}

Tissues were disrupted using lysis beads and a homogenizer unit (Precellys, UK) in RLT buffer (Qiagen, UK). Sorted or cultured cells were lysed directly in RLT buffer and homogenized by pipetting. RNA was isolated using RNEasy Mini or Micro kits (Qiagen, UK) followed by reverse transcription using random primers (Applied Biosystems, UK). Quantitative PCR (qPCR) was performed using Taqman assays (Applied Biosystems) and PrecisionPlus Mastermix (Primer Design, UK) on a ViiA7 384-well real-time PCR detection system (Applied Biosystems). All expression levels were normalized to an internal house keeping (HK) gene ( $R P L P O$ for human samples and Hprt for mouse samples) and calculated as $2^{\wedge}-\left(\mathrm{CT}_{\mathrm{HK}}-\mathrm{CT}\right.$ gene $)$.

\section{Flow cytometry and cell sorting}

Mouse cells were stained with combinations of the following monoclonal antibodies according to manufacturer protocols: CD3-PE (UCH-T1), CD4-BV605 (RM4-5), CD8-APC (53-6.7), CD11b-PerCP-Cy5.5 (M1/70), CD11c-efluor450 (N418), CD19-biotin (6D5), CD31-BV605 (390), CD44-V500 (IM7), CD44-AF700 (IM7), CD45-BV650 (30-F11), CD45-AF700 (30-F11), Foxp3-efluor450 (FJK-16s), PDPN-PE-Cy7 (8.1.1), Gr1-APC (RB6-8C5), ICAM1-PE (YN1/1.7.4), Ly6C-PE-Cy7 (HK1.4), MHCII-AF700 (M5/114.15.2), siglec-f-PE (E50-2440), and TCR $\beta-B V 510$ (H57-597). Human cells were stained with the following monoclonal antibodies: CD3-BV510 (OKT3), CD4-PEDazzle594 (RPA-T4), CD19-BV650 (HIB19), CD31-BV605 (WM59), CD45-AF700 (HI30), CD45RA-PE-Cy7 (HI100), EpCAM-FITC (9C4), PDPN-AF647 (NC-08), HLADR-BV711 (L243), ICAM1-BV421 (HA58), OSM-APC (17022), OSMR-PE (AN-V2), TNF-efluor450 (MAb-11), IFN- $\gamma$-FITC (B27), and IL-17A-PE (eBio64DEC17). All antibodies were from eBioscience (UK), Biolegend (UK), Becton Dickinson (UK), or R\&D Systems (UK). Dead cells were excluded using efluor-780 fixable viability dye (eBioscience). Samples were acquired on FACS LSRFortessa and FACS LSRII flow cytometers (Becton Dickinson). Cell sorting was performed using a FACS ARIA III (Becton 
Dickinson). Data were analyzed using FlowJo (Tree Star, USA). For intracellular cytokine staining, cells were restimulated with PMA (5 ng/ml; Sigma-Aldrich), ionomycin (500 $\mathrm{ng} / \mathrm{ml}$; Sigma-Aldrich), and $5 \mu \mathrm{g} / \mathrm{ml}$ brefeldin A (Sigma-Aldrich). After 4 hours, cells were stained with fixable viability dye and surface markers, fixed with $2 \%$ formaldehyde (Merck, UK), and stained for intracellular cytokines in permeabilization buffer containing $0.05 \%$ saponin (Sigma-Aldrich). For staining Foxp3, cells were stained with fixable viability dye and surface markers prior to fixation and permeabilization using the Foxp3 staining buffer kit (eBioscience) according to manufacturer instructions.

\section{OSMR staining}

To stain OSMR in human samples for flow cytometry analysis, cells were labeled with primary OSMR-PE antibody (clone AN-V2, $2 \mu \mathrm{g} / \mathrm{ml}$ ) followed by three rounds of amplification with anti-PE-biotin antibody (clone PE001, $2.5 \mu \mathrm{g} / \mathrm{ml}$ (Biolegend, UK)), and streptavidin-PE $(0.4 \mu \mathrm{g} / \mathrm{ml}$ (Biolegend, UK)). A separate cell sample was labeled with isotype control antibody (mouse IgG1-PE) and similarly amplified to control for background staining. Specificity of the anti-OSMR antibody was confirmed by siRNA knockdown of OSMR expression in a prior publication65.

\section{Immunohistochemistry}

Formalin-fixed paraffin-embedded tissues were sectioned at five microns and collected onto Superfrost glass slides. Tissue sections were dewaxed in xylene and rehydrated through graded alcohol to water. Endogenous peroxidase activity was blocked with $3 \%(\mathrm{v} / \mathrm{v})$ hydrogen peroxide before masked antigens were retrieved by microwaving the tissue sections in target retrieval solution (Dako). Endogenous avidin and biotin were blocked (Vector Laboratories) and the tissue sections blocked with $10 \%(\mathrm{v} / \mathrm{v})$ normal horse serum (Sigma Aldrich). Human tissue sections were incubated overnight at $4^{\circ} \mathrm{C}$ in a humidified environment with monoclonal mouse anti-PDPN antibody (Clone D2-40; Dako). Primary labelling was detected using biotinylated horse anti-mouse $\operatorname{IgG}$ secondary antibody (Vector Laboratories). Tissue sections were then incubated with streptavidin-horseradish peroxidase (Vector Laboratories) and signal detected using diaminobenzidine (Vector Laboratories). Tissue sections were counterstained with Mayer's Haematoxylin (Sigma Aldrich) before being dehydrated through graded alcohol to xylene and mounted with DPX and coverslips applied. Mouse tissues were embedded into paraffin wax, sectioned, and antigens unmasked as above. Tissue sections were labelled with APC-conjugated hamster anti-mouse PDPN (clone 8.1.1; Biolegend) or APC-conjugated IgG isotype control, before being counterstained with Hoechst 33258 and mounted in N-propyl gallate in glycerol-PBS. Images were collected on an Olympus BX51 microscope. PDPN was chosen as a stromal marker for immunohistochemistry for several reasons, including (a) anti-PDPN antibodies display excellent signal to noise ratio on formalin-fixed tissue sections; (b) PDPN staining is clearly specific for stromal and lymphatic endothelial cells in mouse and human based on flow cytometry analysis; and (c) the immunohistochemical performance of anti-PDPN staining can be easily confirmed by observing staining of endothelial vessels.

For detection of mouse CD3, B220, and F4/80, tissue samples were fixed in formalin and embedded in paraffin. Paraffin sections were dewaxed and stained with hematoxylin and 
eosin (H\&E) for overview. For immunohistochemistry, the sections were incubated with anti-B220 (clone RA3-6B2, eBioscience) followed by incubation with secondary antibody (rabbit anti-rat, Dako). For detection, EnVision+ System-HRP Labelled Polymer AntiRabbit (Dako) was used. HRP was visualized with the chromogen diaminobenzidine (Dako). After color development, sections were subjected to a heat-induced epitope retrieval step prior to incubation with anti-CD3 $\varepsilon$ antibody (clone M-20, Santa Cruz) followed by incubation with biotinylated secondary antibody (Dianova). For detection, alkaline phosphatase-labelled streptavidin and chromogen RED (both Dako) were employed. For the detection of macrophages, sections were subjected to protein-induced epitope retrieval employing protease (Sigma) prior to incubation with anti-F4/80 (clone BM8, eBioscience) followed by incubation with biotinylated rabbit anti-rat secondary antibody (Dako). Biotin was detected using alkaline phosphatase-labelled streptavidin (Dako). For visualization of alkaline phosphatase, chromogen RED (Dako) was used. Negative controls were performed by omitting the primary antibody, and nuclei were stained with hematoxylin. Sections were coverslipped with glycerol gelatin (Merck).

\section{Osmr detection by in situ hybridization}

For detection of mouse Osmr mRNA, the RNAScope ${ }^{\circledR} 2.5$ HD Reagent Kit-RED (ACD Europe SRL) was used. Briefly, paraffin sections were freshly cut, dried for 1 hour at $60^{\circ} \mathrm{C}$ and dewaxed prior to mild unmasking with Target Retrieval buffer and protease. Pretreated sections were hybridized with specific probes to Omsr and Ppib (positive control) and irrelevant probe to $d a p b$ as a negative control. These were accompanied by an additional slide with formalin-fixed and paraffin embedded 3T3 cell line as an additional positive control. After hybridization signal amplification, binding of probes was visualized using FastRed. Nuclei were stained with hematoxylin and sections were coverslipped with Ecomount.

Images were acquired using the AxioImager Z1 microscope (Carl Zeiss MicroImaging). All evaluations were performed in a blinded manner. For final presentation, images were enhanced using Adobe Photoshop to increase contrast between the red Osmr signal and background hematoxylin.

\section{Total protein extraction and immunoblot analysis}

Total protein extracts were prepared as described66. Equal protein amounts were resolved by SDS-PAGE and analyzed with anti-p-STAT3 (D3A7), anti-p-STAT1 (D4A7), anti-p-ERK1/2 (D13.14.4E), anti-p-AKT (D9E), anti-p-p38 (D3F9), and anti- $\beta$-actin (13E5). All antibodies were from Cell Signaling, UK.

\section{Statistical analysis}

Unless otherwise indicated, all bar charts represent means +/- S.E.M. Parametric and nonparametric analyses were used where appropriate based on testing for a normal distribution using the D'Agostino-Pearson Omnibus normality test. Statistical tests were two-sided and specified in figure legends. Differences were considered to be significant when $p<0.05$. Multiple testing corrections were applied where appropriate. In rare situations, data points 
were excluded from analysis only if they were found to be outliers using the ROUT method at $\mathrm{Q}=1 \%$ (pre-determined criteria).

\section{Supplementary Material}

Refer to Web version on PubMed Central for supplementary material.

\section{Acknowledgements}

We thank Jenny Middleton, Sebastian Rogatti Granados, Dr. Carolina Arancibia, Priya Siddhanathi, James Chivenga, Ngonidzashe Charumbira, Dr. Julie Schulthess, and Dr. Claire Pearson, and Simone Spieckermann for excellent technical support, patient sample collection, and lab management. We thank Dr. Holm Uhlig and all members of the laboratory for valuable discussions and support. We also thank Dr. Vivianne Malmstrom and Dr. Elizabeth Thompson for critical review of this manuscript. We gratefully acknowledge the contributions of the Oxford Radcliffe and GI Biobanks and the Oxford IBD cohort study, which are supported by the NIHR Oxford Biomedical Research Centre (grant no. HBRWAE04 Task HB81.G). We thank the flow cytometry core facility staff at the University of Oxford Translational Gastroenterology Unit and Kennedy Institute of Rheumatology, as well as the staff of the Kennedy Institute histology core facility for excellent tissue preparation. We thank all volunteers, patients, and their families for agreeing to contribute to this study. NRW was supported by an Irvington Institute Post-doctoral Fellowship (Cancer Research Institute). ANH was supported by a European Molecular Biology Organization (EMBO) long-term fellowship (ALTF 116-2012) and a Marie Curie fellowship (FP7-PEOPLE-2012IEF, Proposal 330621). BMJO is a Lecturer at Somerville College, University of Oxford. ST was supported by the Ecole Normale Supérieure of Lyon and the French Ministry of Education. RJO and NR are supported by Medical Research Council grant no. MR/K018779/1. MC and KJM were supported by the Edward Penley Abraham Trust. KJM is supported by the Wellcome Trust (Investigator Award 102972). FP is supported by the Wellcome Trust and Foundation Louis Jeantet.

\section{References}

1. Uniken Venema WT, Voskuil MD, Dijkstra G, Weersma RK, Festen EA. The genetic background of inflammatory bowel disease: from correlation to causality. J Pathol. 2017; 241:146-158. [PubMed: 27785786]

2. Basson A, Trotter A, Rodriguez-Palacios A, Cominelli F. Mucosal Interactions between Genetics, Diet, and Microbiome in Inflammatory Bowel Disease. Front Immunol. 2016; 7:290. [PubMed: 27531998]

3. de Souza HSP, Fiocchi C. Immunopathogenesis of IBD: current state of the art. Nat Rev Gastroenterol Hepatol. 2016; 13:13-27. [PubMed: 26627550]

4. Maloy KJ, Powrie F. Intestinal homeostasis and its breakdown in inflammatory bowel disease. Nature. 2011; 474:298-306. [PubMed: 21677746]

5. Neurath MF. Cytokines in inflammatory bowel disease. Nat Rev Immunol. 2014; 14:329-342. [PubMed: 24751956]

6. Chen ML, Sundrud MS. Cytokine Networks and T-Cell Subsets in Inflammatory Bowel Diseases. Inflammatory Bowel Diseases. 2016; 22:1157-1167. [PubMed: 26863267]

7. Ben-Horin S, Chowers Y. Tailoring anti-TNF therapy in IBD: drug levels and disease activity. Nat Rev Gastroenterol Hepatol. 2014; 11:243-255. [PubMed: 24393836]

8. Bermejo F, Guerra I. Management of inflammatory bowel disease in poor responders to infliximab. CEG. 2014; :359-9. DOI: 10.2147/CEG.S45297

9. Hueber W, et al. Secukinumab, a human anti-IL-17A monoclonal antibody, for moderate to severe Crohn's disease: unexpected results of a randomised, double-blind placebo-controlled trial. Gut. 2012; 61:1693-1700. [PubMed: 22595313]

10. Garbers C, et al. Plasticity and cross-talk of interleukin 6-type cytokines. Cytokine \& Growth Factor Reviews. 2012; 23:85-97. [PubMed: 22595692]

11. Richards CD. The enigmatic cytokine oncostatin $\mathrm{m}$ and roles in disease. ISRN Inflammation. 2013; 2013:512103-23. [PubMed: 24381786]

12. Hermanns HM. Oncostatin M and interleukin-31: Cytokines, receptors, signal transduction and physiology. Cytokine \& Growth Factor Reviews. 2015; 26:545-558. [PubMed: 26198770] 
13. Jostins L, et al. Host-microbe interactions have shaped the genetic architecture of inflammatory bowel disease. Nature. 2012; 491:119-124. [PubMed: 23128233]

14. Beigel F, et al. Oncostatin M Mediates STAT3-Dependent Intestinal Epithelial Restitution via Increased Cell Proliferation, Decreased Apoptosis and Upregulation of SERPIN Family Members. PLoS ONE. 2014; 9:e93498-15. [PubMed: 24710357]

15. Sanchez AL, et al. Adenoviral transfer of the murine oncostatin M gene suppresses dextran-sodium sulfate-induced colitis. J Interferon Cytokine Res. 2003; 23:193-201. [PubMed: 12856331]

16. Haberman Y, et al. Pediatric Crohn disease patients exhibit specific ileal transcriptome and microbiome signature. J Clin Invest. 2014; 124:3617-3633. [PubMed: 25003194]

17. Vanhove W, et al. Strong Upregulation of AIM2 and IFI16 Inflammasomes in the Mucosa of Patients with Active Inflammatory Bowel Disease. Inflammatory Bowel Diseases. 2015; 21:26732682. [PubMed: 26313692]

18. Arijs I, et al. Mucosal Gene Expression of Antimicrobial Peptides in Inflammatory Bowel Disease Before and After First Infliximab Treatment. PLoS ONE. 2009; 4:e7984-10. [PubMed: 19956723]

19. Galamb O, et al. Reversal of gene expression changes in the colorectal normal-adenoma pathway by NS398 selective COX2 inhibitor. Br J Cancer. 2010; 102:765-773. [PubMed: 20087348]

20. Planell N, et al. Transcriptional analysis of the intestinal mucosa of patients with ulcerative colitis in remission reveals lasting epithelial cell alterations. Gut. 2013; 62:967-976. [PubMed: 23135761]

21. D'Haens GR, et al. The London Position Statement of the World Congress of Gastroenterology on Biological Therapy for IBD with the European Crohn's and Colitis Organization: when to start, when to stop, which drug to choose, and how to predict response? Am J Gastroenterology. 2011; 106:199-212. quiz 213.

22. Arijs I, et al. Mucosal gene signatures to predict response to infliximab in patients with ulcerative colitis. Gut. 2009; 58:1612-1619. [PubMed: 19700435]

23. Toedter G, et al. Gene expression profiling and response signatures associated with differential responses to infliximab treatment in ulcerative colitis. Am J Gastroenterology. 2011; 106:12721280.

24. Sandborn WJ, et al. Colectomy rate comparison after treatment of ulcerative colitis with placebo or infliximab. Gastroenterology. 2009; 137:1250-60. quiz 1520. [PubMed: 19596014]

25. Sandborn WJ, et al. Subcutaneous golimumab induces clinical response and remission in patients with moderate-to-severe ulcerative colitis. Gastroenterology. 2014; 146:85-95. quiz e14-5. [PubMed: 23735746]

26. Bindea G, et al. ClueGO: a Cytoscape plug-in to decipher functionally grouped gene ontology and pathway annotation networks. Bioinformatics. 2009; 25:1091-1093. [PubMed: 19237447]

27. Owens BMJ, et al. CD90(+) Stromal Cells are Non-Professional Innate Immune Effectors of the Human Colonic Mucosa. Front Immunol. 2013; 4:307. [PubMed: 24137162]

28. Owens BMJ. Inflammation, Innate Immunity, and the Intestinal Stromal Cell Niche: Opportunities and Challenges. Front Immunol. 2015; 6:1-7. [PubMed: 25657648]

29. Vicente-Suarez I, et al. Unique lamina propria stromal cells imprint the functional phenotype of mucosal dendritic cells. Mucosal Immunol. 2015; 8:141-151. [PubMed: 24938743]

30. Peduto L, et al. Inflammation recapitulates the ontogeny of lymphoid stromal cells. J Immunol. 2009; 182:5789-5799. [PubMed: 19380827]

31. Baptista AP, et al. Colonic patch and colonic SILT development are independent and differentially regulated events. Mucosal Immunol. 2013; 6:511-521. [PubMed: 22990625]

32. Astarita JL, Acton SE, Turley SJ. Podoplanin: emerging functions in development, the immune system, and cancer. Front Immunol. 2012; 3:283. [PubMed: 22988448]

33. Fletcher AL, Acton SE, Knoblich K. Lymph node fibroblastic reticular cells in health and disease. Nat Rev Immunol. 2015; 15:350-361. [PubMed: 25998961]

34. Kullberg MC, et al. IL-23 plays a key role in Helicobacter hepaticus-induced T cell-dependent colitis. Journal of Experimental Medicine. 2006; 203:2485-2494. [PubMed: 17030948]

35. Schiering C, et al. The alarmin IL-33 promotes regulatory T-cell function in the intestine. Nature. 2014; 513:564-568. [PubMed: 25043027] 
36. Kullberg MC, et al. Helicobacter hepaticus-induced colitis in interleukin-10-deficient mice: cytokine requirements for the induction and maintenance of intestinal inflammation. Infect Immun. 2001; 69:4232-4241. [PubMed: 11401959]

37. Arnold IC, et al. CD11c+ monocyte/macrophages promote chronic Helicobacter hepaticus-induced intestinal inflammation through the production of IL-23. Mucosal Immunol. 2015; doi: 10.1038/mi.2015.65

38. Brolund L, Küster A, Korr S, Vogt M, Müller-Newen G. A receptor fusion protein for the inhibition of murine oncostatin M. BMC Biotechnol. 2011; 11:3. [PubMed: 21223559]

39. Feagan BG, et al. Ustekinumab as Induction and Maintenance Therapy for Crohn's Disease. N Engl J Med. 2016; 375:1946-1960. [PubMed: 27959607]

40. Ryan RE, et al. Oncostatin M binds to extracellular matrix in a bioactive conformation: implications for inflammation and metastasis. Cytokine. 2015; 72:71-85. [PubMed: 25622278]

41. Bottini N, Firestein GS. Duality of fibroblast-like synoviocytes in RA: passive responders and imprinted aggressors. Nat Rev Rheumatol. 2013; 9:24-33. [PubMed: 23147896]

42. McLean LP, Cross RK. Adverse events in IBD: to stop or continue immune suppressant and biologic treatment. Expert Rev Gastroenterol Hepatol. 2014; 8:223-240. [PubMed: 24490595]

43. Murdaca G, et al. Infection risk associated with anti-TNF-a agents: a review. Expert Opin Drug Saf. 2015; 14:571-582. [PubMed: 25630559]

44. Komori T, Tanaka M, Senba E, Miyajima A, Morikawa Y. Deficiency of oncostatin M receptor $\beta$ (OSMR $\beta$ ) exacerbates high-fat diet-induced obesity and related metabolic disorders in mice. J Biol Chem. 2014; 289:13821-13837. [PubMed: 24695736]

45. Hamada T, et al. Oncostatin M gene therapy attenuates liver damage induced by dimethylnitrosamine in rats. The American Journal of Pathology. 2007; 171:872-881. [PubMed: 17640959]

46. Nakamura K, Tanaka M, Saito H, Nonaka H, Miyajima A. Hepatocyte proliferation and tissue remodeling is impaired after liver injury in oncostatin $\mathrm{M}$ receptor knockout mice. Hepatology. 2004; 39:635-644. [PubMed: 14999682]

47. Kubin $\mathrm{T}$, et al. Oncostatin $\mathrm{M}$ is a major mediator of cardiomyocyte dedifferentiation and remodeling. Cell Stem Cell. 2011; 9:420-432. [PubMed: 22056139]

48. Pöling $J$, et al. Therapeutic targeting of the oncostatin M receptor- $\beta$ prevents inflammatory heart failure. Basic Res Cardiol. 2014; 109:396-14. [PubMed: 24292852]

49. Choy EH, et al. Safety, tolerability, pharmacokinetics and pharmacodynamics of an antion-costatin $\mathrm{M}$ monoclonal antibody in rheumatoid arthritis: results from phase II randomized, placebocontrolled trials. Arthritis Res Ther. 2013; 15:R132. [PubMed: 24286335]

50. Tanaka M, Hirabayashi Y. Targeted disruption of oncostatin M receptor results in altered hematopoiesis. 2003; 102:3154-3162.

51. Morikawa $\mathrm{Y}$, et al. Essential function of oncostatin $\mathrm{m}$ in nociceptive neurons of dorsal root ganglia. J Neurosci. 2004; 24:1941-1947. [PubMed: 14985435]

52. Pohin M, et al. Oncostatin M overexpression induces skin inflammation but is not required in the mouse model of imiquimod-induced psoriasis-like inflammation. Eur J Immunol. 2016; 46:17371751. [PubMed: 27122058]

53. Guilloteau K, et al. Skin Inflammation Induced by the Synergistic Action of IL-17A, IL-22, Oncostatin M, IL-1 $\{$ alpha\}, and TNF-\{alpha\} Recapitulates Some Features of Psoriasis. J Immunol. 2010; 184:5263-5270.

54. Gazel A, et al. A characteristic subset of psoriasis-associated genes is induced by oncostatin-M in reconstituted epidermis. J Invest Dermatol. 2006; 126:2647-2657. [PubMed: 16917497]

55. Boniface $\mathrm{K}$, et al. Oncostatin $\mathrm{M}$ secreted by skin infiltrating T lymphocytes is a potent keratinocyte activator involved in skin inflammation. The Journal of Immunology. 2007; 178:4615-4622. [PubMed: 17372020]

56. Langdon $\mathrm{C}$, et al. Murine oncostatin $\mathrm{M}$ stimulates mouse synovial fibroblasts in vitro and induces inflammation and destruction in mouse joints in vivo. The American Journal of Pathology. 2000; 157:1187-1196. [PubMed: 11021823] 
57. Hui W, Richards CD, Cawston TE, Rowan AD. A model of inflammatory arthritis highlights a role for oncostatin $M$ in pro-inflammatory cytokine-induced bone destruction via RANK/RANKL. Arthritis Res Ther. 2005; 7:R57-64. [PubMed: 15642143]

58. Hui W, Richards CD, Rowan AD, Cawston TE. Oncostatin M in combination with tumor necrosis factor alpha induces cartilage damage and matrix metalloproteinase expression in vitro and in vivo. Arthritis Rheum. 2003; 48:3404-3418. [PubMed: 14673992]

59. Hintzen C, Heinrich PC, Quaiser S, Pap T, Hermanns HM. Induction of CCL13 expression in synovial fibroblasts highlights a significant role of oncostatin $\mathrm{M}$ in rheumatoid arthritis. Arthritis Rheum. 2009; 60:1932-1943. [PubMed: 19565514]

60. Ott C, Schölmerich J. Extraintestinal manifestations and complications in IBD. Nat Rev Gastroenterol Hepatol. 2013; 10:585-595. [PubMed: 23835489]

61. Geremia A, et al. IL-23-responsive innate lymphoid cells are increased in inflammatory bowel disease. J Exp Med. 2011; 208:1127-1133. [PubMed: 21576383]

62. Becker C, Fantini MC, Neurath MF. High resolution colonoscopy in live mice. Nat Protoc. 2007; 1:2900-2904.

63. Izcue A, et al. Interleukin-23 restrains regulatory $\mathrm{T}$ cell activity to drive $\mathrm{T}$ cell-dependent colitis. Immunity. 2008; 28:559-570. [PubMed: 18400195]

64. Uhlig HH, et al. Characterization of Foxp3+CD4+CD25+ and IL-10-secreting CD4+CD25+ T cells during cure of colitis. The Journal of Immunology. 2006; 177:5852-5860. [PubMed: 17056509]

65. West NR, Murray JI, Watson PH. Oncostatin-M promotes phenotypic changes associated with mesenchymal and stem cell-like differentiation in breast cancer. Oncogene. 2014; 33:1485-1494. [PubMed: 23584474]

66. West NR, Murphy LC, Watson PH. Oncostatin M suppresses oestrogen receptor-a expression and is associated with poor outcome in human breast cancer. Endocrine Related Cancer. 2012; 19:181195. [PubMed: 22267707] 
a
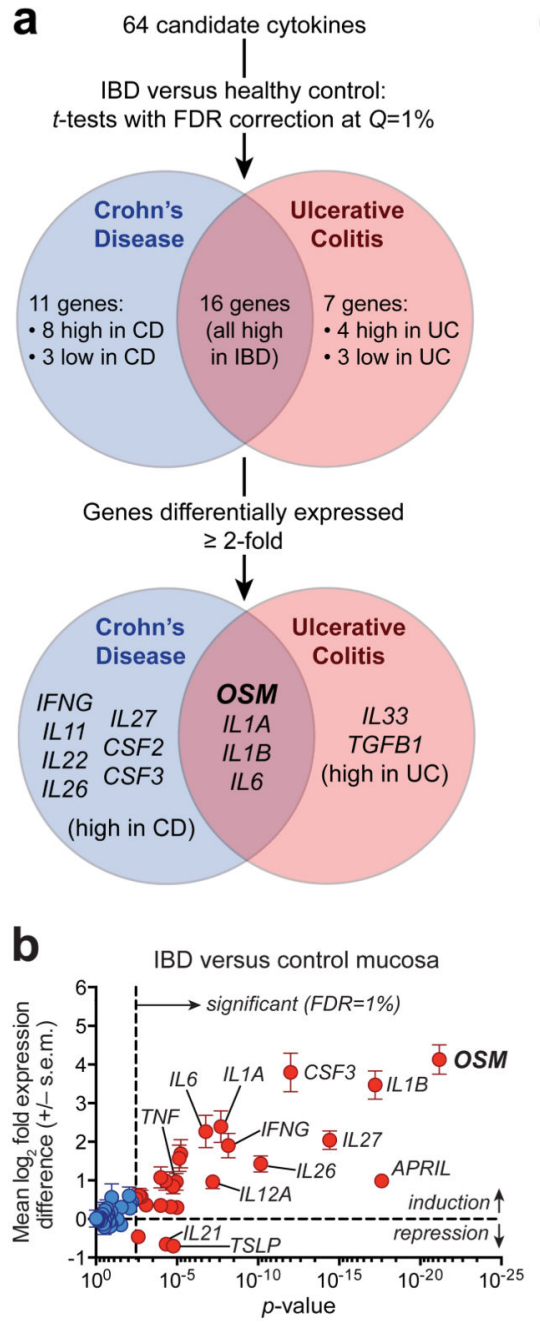

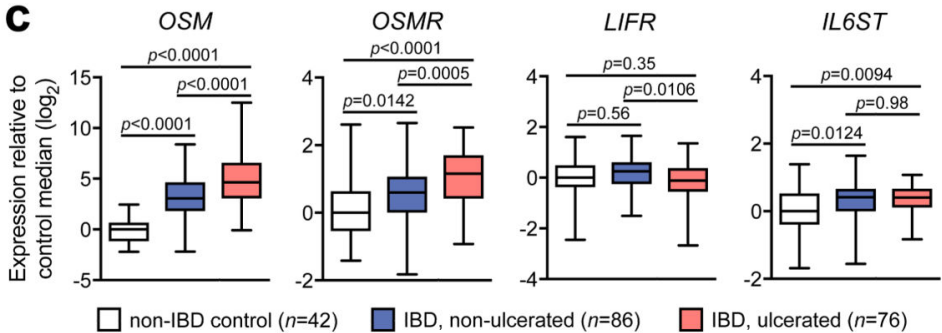

d
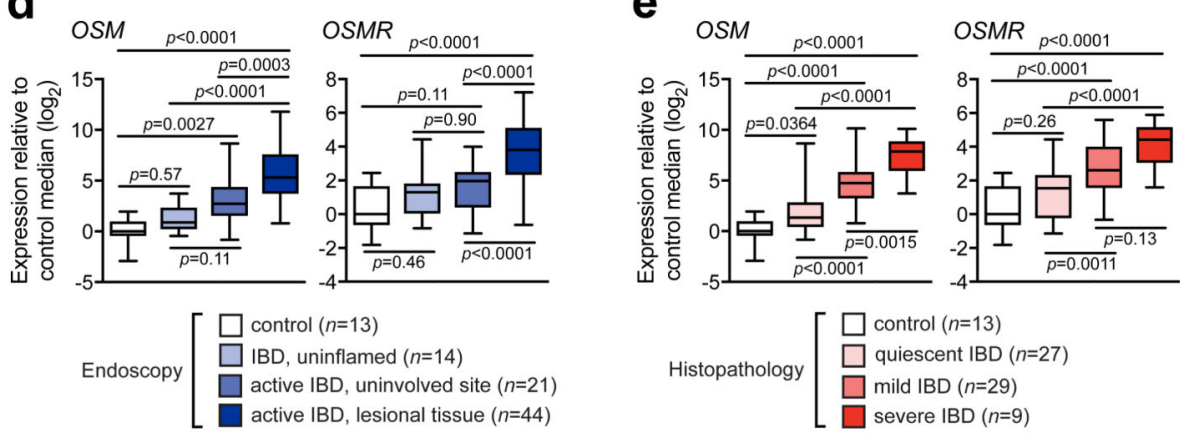

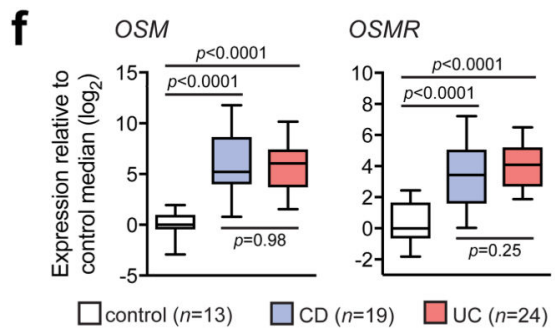

Figure 1. Expression of OSM and OSMR in the inflamed intestinal tissue of patients with IBD.

(a) Identification of cytokines associated with intestinal inflammation in CD and UC patients. Data were derived from Gene Expression Omnibus (GEO) datasets GSE57945 ( $n=42$ controls and $n=162 \mathrm{CD}$ ) and GSE59071 ( $n=11$ controls and $n=74$ UC). Briefly, mRNA expression of 64 cytokines was compared in IBD versus healthy control intestinal tissue using $t$-tests with false discovery rate correction $(\mathrm{Q}=1 \%)$. Significant hits were further selected using a fold difference threshold of $\geq 2$. (b) RNA sequencing analysis of 64 cytokine genes in pediatric treatment-naïve CD patients $(n=162)$ versus non-IBD controls $(n=42$; GEO \#GSE57945). Blue symbols, not statistically significant after $t$-tests with FDR correction $(\mathrm{Q}=1 \%)$; red symbols, significantly altered cytokines. (c) Expression of $O S M$, OSMR, LIFR, and IL6ST (gp130) in the GSE57945 dataset. Statistics: one-way ANOVA with Tukey's multiple comparisons tests $(\mathrm{df}=201) .(\mathbf{d}-\mathbf{f}) \mathrm{Q}-\mathrm{PCR}$ analysis of $O S M$ and OSMR in intestinal mucosal biopsies from IBD patients and healthy controls (Oxford cohort, see Supplementary Table 2 for details). Statistical comparisons made using one-way ANOVA with Tukey's multiple comparisons tests $(\mathrm{df}=88(\mathbf{d}) ; \mathrm{df}=74(\mathbf{e}) ; \mathrm{df}=53(\mathbf{f})) .(\mathbf{d})$ Specimens categorized by macroscopic evidence of disease activity determined during 
endoscopy (includes IBD patients with no macroscopic inflammation (uninflamed), uninflamed specimens from patients with inflammation elsewhere in the bowel (uninvolved), and macroscopically inflamed specimens (lesional tissue)). (e) Samples categorized by inflammation severity, determined by routine clinical histopathological assessment of matched biopsies. (f) Analysis of inflamed lesions from active CD or UC. 
a

e b

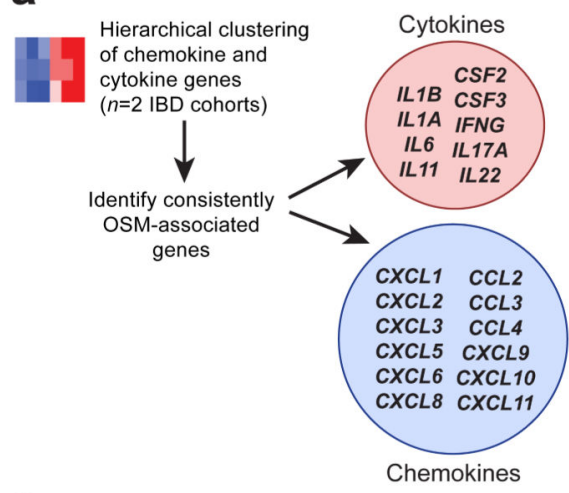

$\square$ UC, remission ( $n=8$ pre, $n=6$ post)

UC, partial response ( $n=15$ pre, $n=11$ post)

$\cup \mathrm{C}$, refractory ( $n=7$ pre, $n=6$ post)
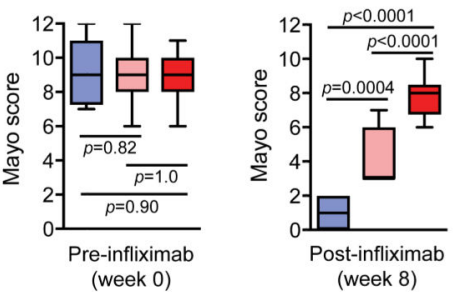

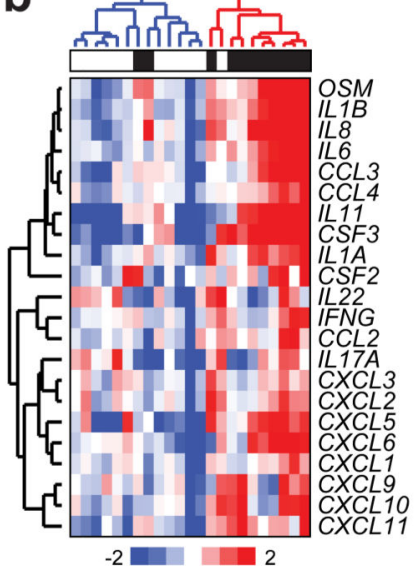

f

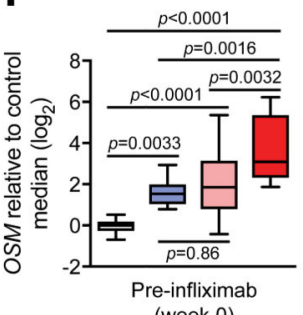

(week 0)
C

$\square$ Infliximab

Infliximab

refractory
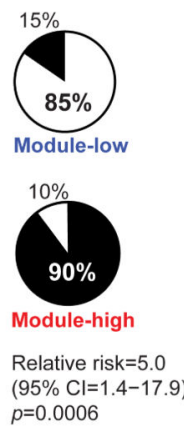

$(95 \% \mathrm{Cl}=1.4-17.9)$

$p=0.0006$
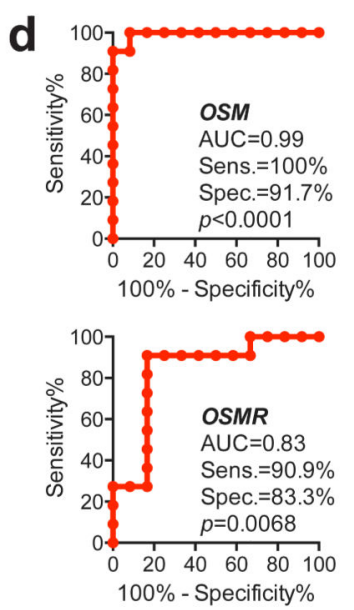

g

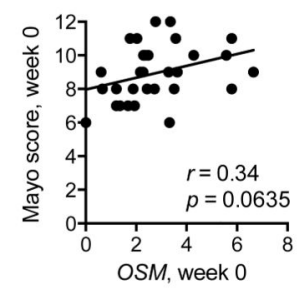

$\square$ healthy controls ( $n=21) \quad \square \cup C$, partial response ( $n=15$ pre, $n=11$ post)

$\square$ UC, remission ( $n=8$ pre, $n=6$ post) $\square$ UC, refractory ( $n=7$ pre, $n=6$ post)

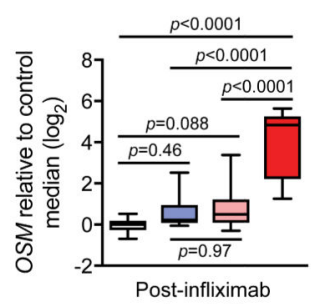

t-infliximab

Figure 2. Association of OSM with response to anti-TNF therapy.

(a) Identification of an OSM-associated inflammatory module in IBD. Expression of genes encoding chemokines and cytokines was examined in two cohorts, including healthy controls and patients with CD or UC (see Supplementary Fig. 2). Samples were grouped using unsupervised hierarchical clustering and 21 genes that correlated closely with OSM in both cohorts were identified. (b) Unsupervised hierarchical clustering of OSM-associated module genes in colonic biopsies of UC patients refractory to corticosteroids or immunosuppression prior to infliximab therapy (GEO \#GSE12251). Patients form two groups after clustering: one with low (blue dendrogram) and one with high OSM-associated module expression (red dendrogram). Patients with complete histological and endoscopic disease resolution are indicated in white, while non-responsive patients are indicated in black (see bar below dendrograms). Data are median-normalized and $\log _{2}$ transformed. Rates of infliximab response in these patients are shown in panel (c). Statistics: Fisher's exact test. (d) Receiver operator characteristic analysis of OSM and OSMR expression, distinguishing infliximab responders and non-responders in the GSE12251 dataset. (e-g) An independent cohort of patients with moderate-to-severe UC treated with infliximab as part of a long-term safety study (clinical trial NCT00207688). (e and f) Mayo scores and colonic $O S M$ expression at baseline and 8 weeks after therapy in different response groups (see Methods for definition of clinical response). Statistics: one-way ANOVA with Tukey's multiple comparisons tests $(\mathrm{df}=27$ (e) and $\mathrm{df}=47(\mathbf{f}))$. (g) Pearson correlation of baseline OSM expression (relative units) and Mayo score. 
a
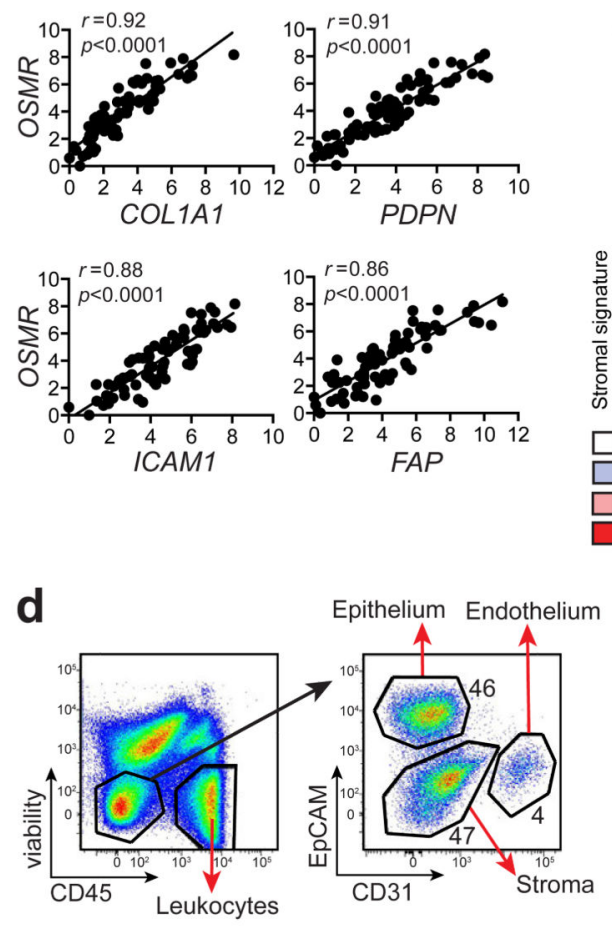

g

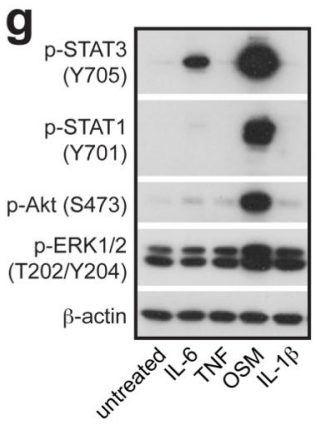

b

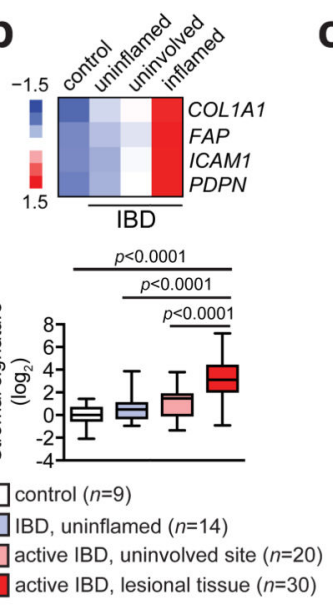

C

PDPN
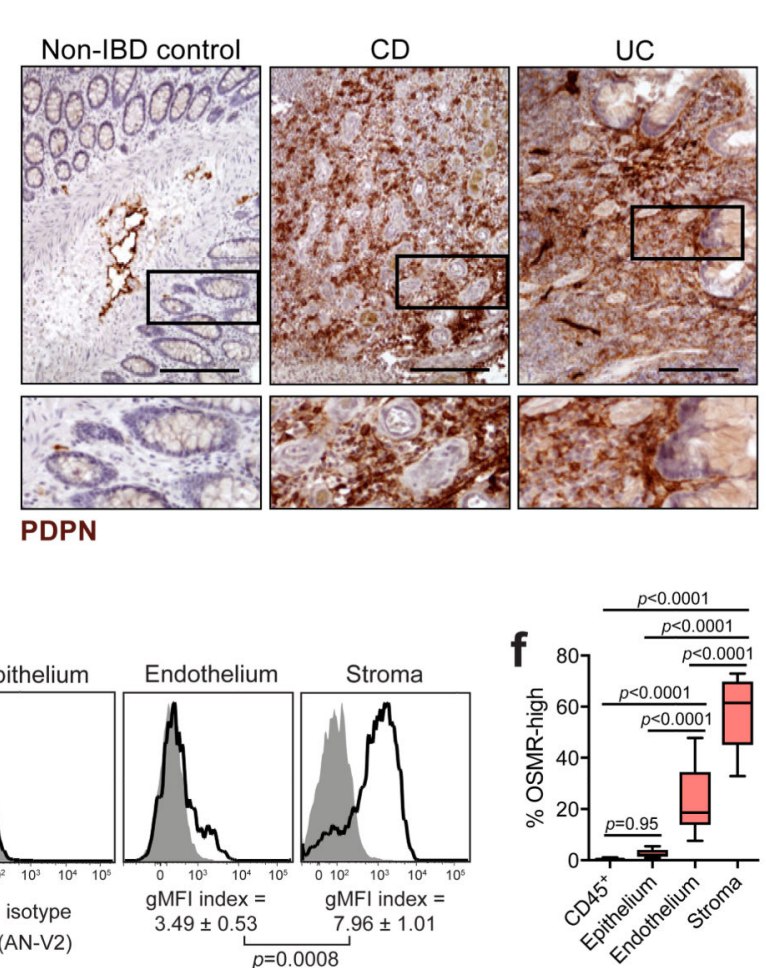

e

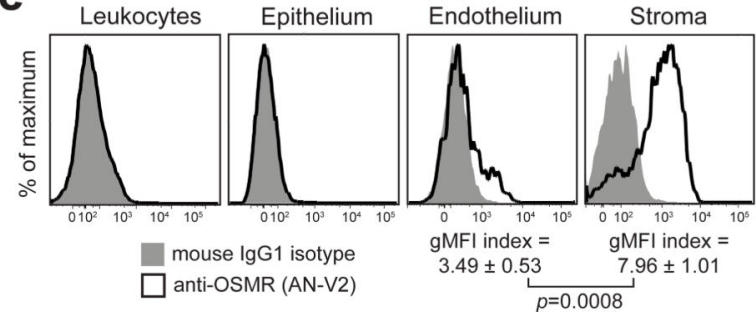

h

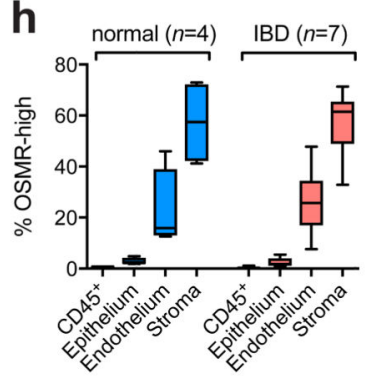

i

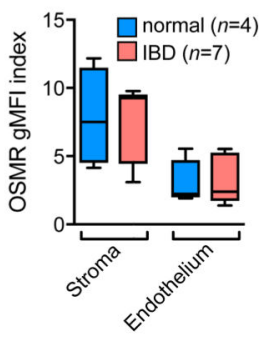

j

Gated on:

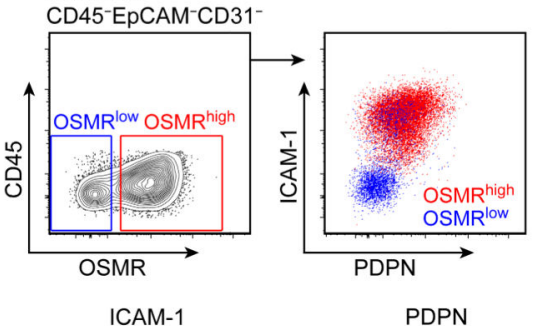

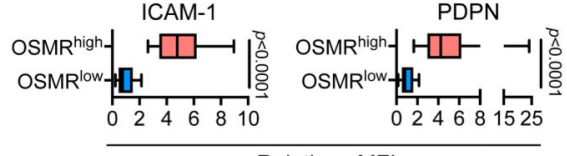

Relative gMFI

Figure 3. Non-hematopoietic stromal cells are prevalent in inflamed intestinal tissue and express high levels of OSMR.

(a) Spearman correlation of $O S M R$ expression with stromal genes in pooled healthy control and IBD biopsies assessed by Q-PCR (Oxford cohort, $n=73$ ). (b) Mean mRNA z-scores for the indicated stromal genes (top) and stromal signature expression (bottom) in intestinal biopsies from healthy controls or IBD patients (uninflamed, no endoscopic evidence of disease; uninvolved, uninflamed samples from patients with active disease; inflamed, samples from inflamed lesions). The stromal signature represents the average log2 expression of $C O L 1 A 1, F A P, I C A M 1$, and PDPN. Statistics: one-way ANOVA with Tukey's multiple comparisons tests $(\mathrm{df}=69)$. (c) Immunohistochemical staining of PDPN in colon tissue from representative non-IBD control, CD, and UC patients (scale bar $=250 \mu \mathrm{m})$. $(\mathbf{d}-\mathbf{f})$ Flow cytometry analysis of human intestinal mucosa ( $n=11$ donors). (d) Identification of 
leukocytes, epithelial cells, endothelial cells, and stroma. (e) OSMR expression and isotypenormalized geometric mean fluorescence intensity (gMFI). Mean (+/- s.e.m.) gMFI compared using $t$-tests $(t=3.924, \mathrm{df}=20$ ). (f) OSMR expression frequencies compared using one-way ANOVA with Tukey's multiple comparisons tests $(\mathrm{df}=40)$. (g) Cropped Western blot images of cytokine-stimulated CCD18Co cell lysates. (h) OSMR expression frequencies in colon mucosal cell populations from non-IBD controls or patients with IBD. (i) Intensity of OSMR expression on colon endothelial and stromal cells from non-IBD controls or patients with IBD. (j) Representative flow cytometry staining of OSMR, ICAM-1, and PDPN on colon stroma, with ICAM-1 and PDPN gMFIs quantified below. Statistics: Mann-Whitney $U$ tests $(n=11)$. 
a

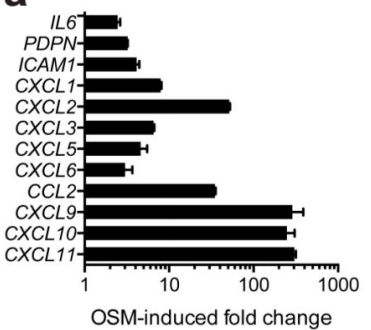

b Gated on CD45-EPCAM- CD31-

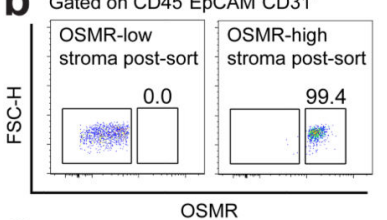

c

OSMR- OSMR-
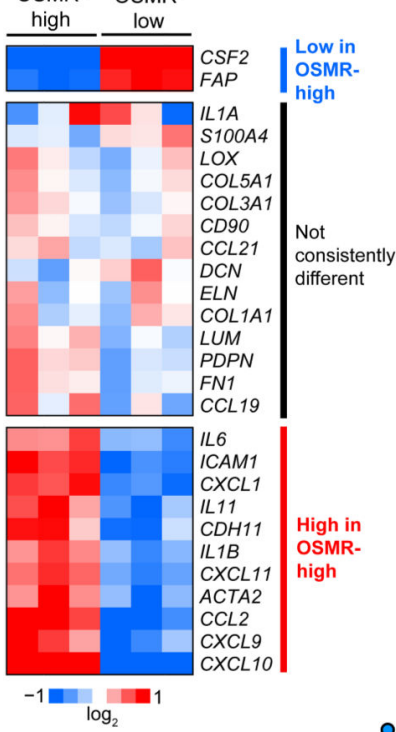

$f$

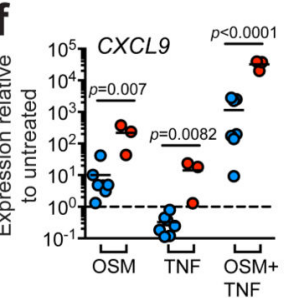

d
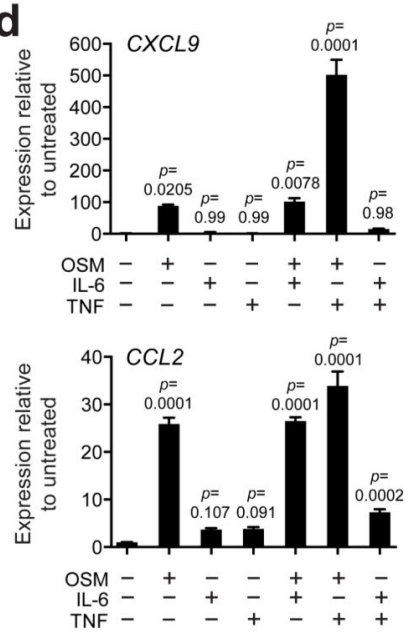

e
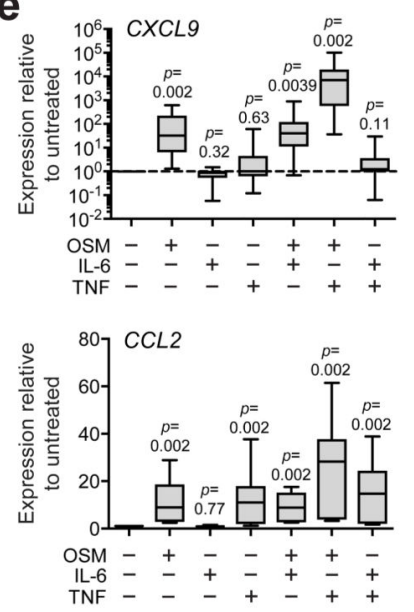

O non-IBD control $(n=7)$

- IBD $(n=3)$

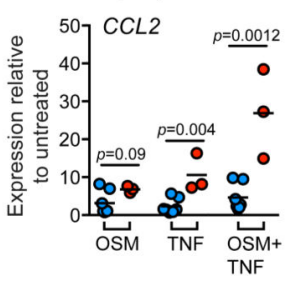

Figure 4. OSM promotes inflammatory behavior in human intestinal stroma.

(a) Q-PCR analysis of OSM-induced genes in triplicate cultures of CCD18Co cells (normal human colonic stroma), relative to untreated conditions. Results are representative of three independent experiments. (b-c) Analysis of OSMR ${ }^{\text {low }}$ and OSMR ${ }^{\text {high }}$ stromal cells purified from resected human intestinal tissue using fluorescence-activated cell sorting (FACS). (b) Post-sorting cell purities from a representative non-IBD control. (c) Q-PCR analysis of stromal and inflammatory genes in the purified stromal fractions from $n=3$ donors. (d) QPCR analysis of triplicate CCD18Co cultures (representative of three independent 
experiments). Cells were stimulated with human OSM, TNF, IL-6, or combinations of the three for 2 hours and compared to untreated controls. Statistics: one-way ANOVA with Dunnett's multiple comparisons tests ( $\mathrm{df}=14$ ). (e) Q-PCR analysis of primary intestinal stromal cultures from 10 donors, stimulated as in panel (d). Data represent fold changes between matched untreated and cytokine-stimulated samples. Statistics: Wilcoxon signed rank test, versus a theoretical median of 1. (f) Q-PCR analysis of $C X C L 9$ and $C C L 2$ expression in cytokine-stimulated stromal cultures from non-IBD controls $(n=7)$ and IBD patients $(n=3)$. Data represent fold changes between matched untreated and cytokinestimulated samples. Statistics: $t$-tests $(\mathrm{df}=8)$. For $C X C L 9, t=3.594$ (OSM), $t=3.493$ (TNF), and $t=8.278$ (OSM+TNF). For $C C L 2, t=1.928$ (OSM), $t=3.940$ (TNF), and $t=4.87$ (OSM $+\mathrm{TNF})$. 
a
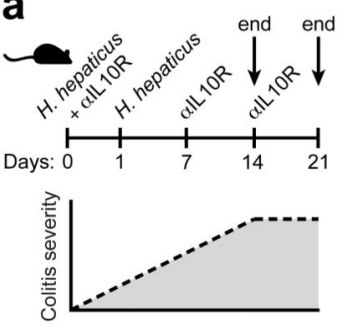

b

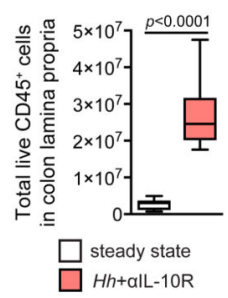

C

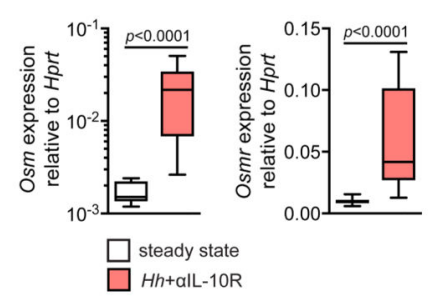

d

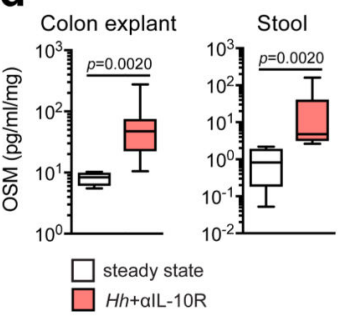

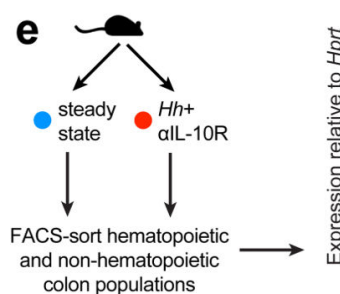
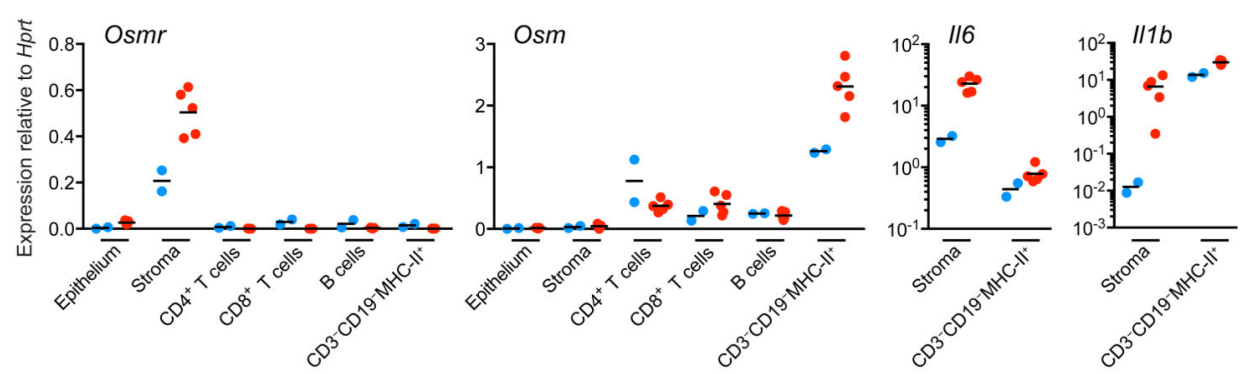

f
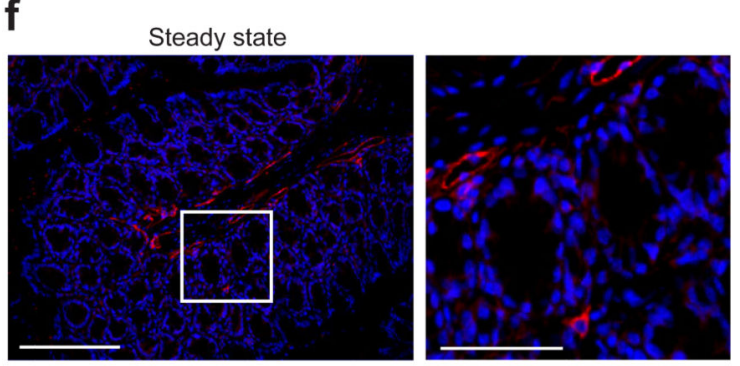

$H h+$ alL-10R colitis, day 21
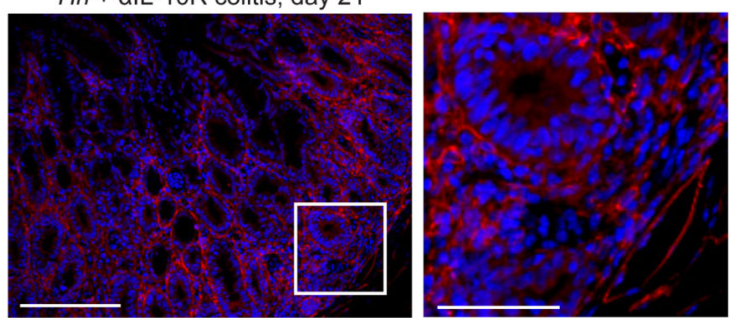

9

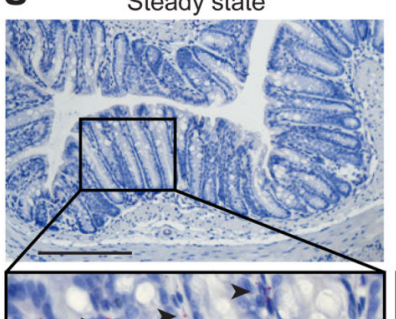

$H h+$ alL-10R colitis (day 14)

Hoechst-33258 PDPN

Hoechst-33258

Hamster IgG

isotype

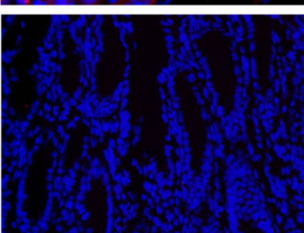

Figure 5. The OSM-stromal cell axis is conserved in anti-TNF resistant murine colitis.

(a) Induction of colitis using the $H h+a$ IL-10R protocol. Mice are sacrificed at day 14 or day 21, which corresponds to peak disease severity. (b) Total live $\mathrm{CD} 45^{+}$cells in the colon lamina propria at day 14 ( $n=8$ steady state and $n=9$ colitic mice, representative of $>3$ independent experiments). (c) Q-PCR analysis of $\mathrm{Osm}$ and $\mathrm{Osmr}$ expression in whole-colon tissue from steady state $(n=8)$ and colitic mice $(n=15)$, representative of $>3$ independent experiments. (d) OSM measured by ELISA in mouse colon explant supernatants and cecal stool extracts from one of two independent experiments (steady state mice, $n=4$; colitic 
mice, $n=10$ ). In panels $\mathbf{b}-\mathbf{d}$, groups are compared using Mann-Whitney $U$-tests. (e) Q-PCR gene expression analysis of FACS-purified populations from mouse colon (representative of two independent experiments). Each data point represents lamina propria cells pooled from two mice. Stromal cells were defined as $\mathrm{CD}^{-} 5^{-} \mathrm{EpCAM}^{-} \mathrm{CD} 31^{-}$. (f) Immunofluorescent detection of PDPN ${ }^{+}$stromal cells in healthy and inflamed mouse colon tissue. Scale bars, $250 \mu \mathrm{m}$ (left) and $100 \mu \mathrm{m}$ (right). (g) Detection of Osmr expression in healthy and inflamed mouse colon tissue using in situ hybridization (punctate red signal). Tissues were counterstained with hematoxylin. Examples of Osmr-expressing cells in healthy tissue are indicated with arrowheads. Scale bars, $250 \mu \mathrm{m}$ (top) and $100 \mu \mathrm{m}$ (bottom). PDPN and Osmr images are representative of 3 different mice per condition. 


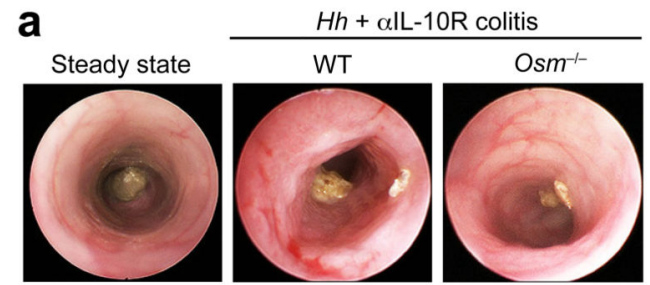

C
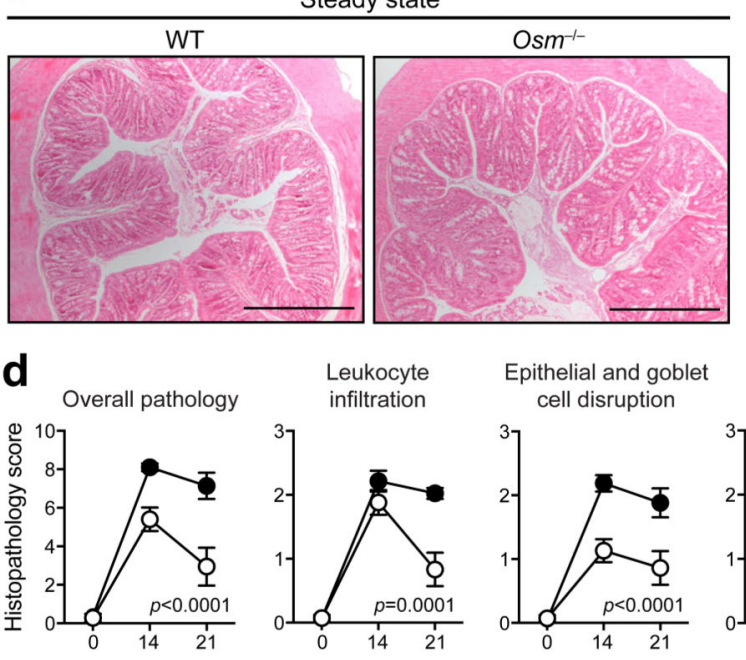

Days of colitis
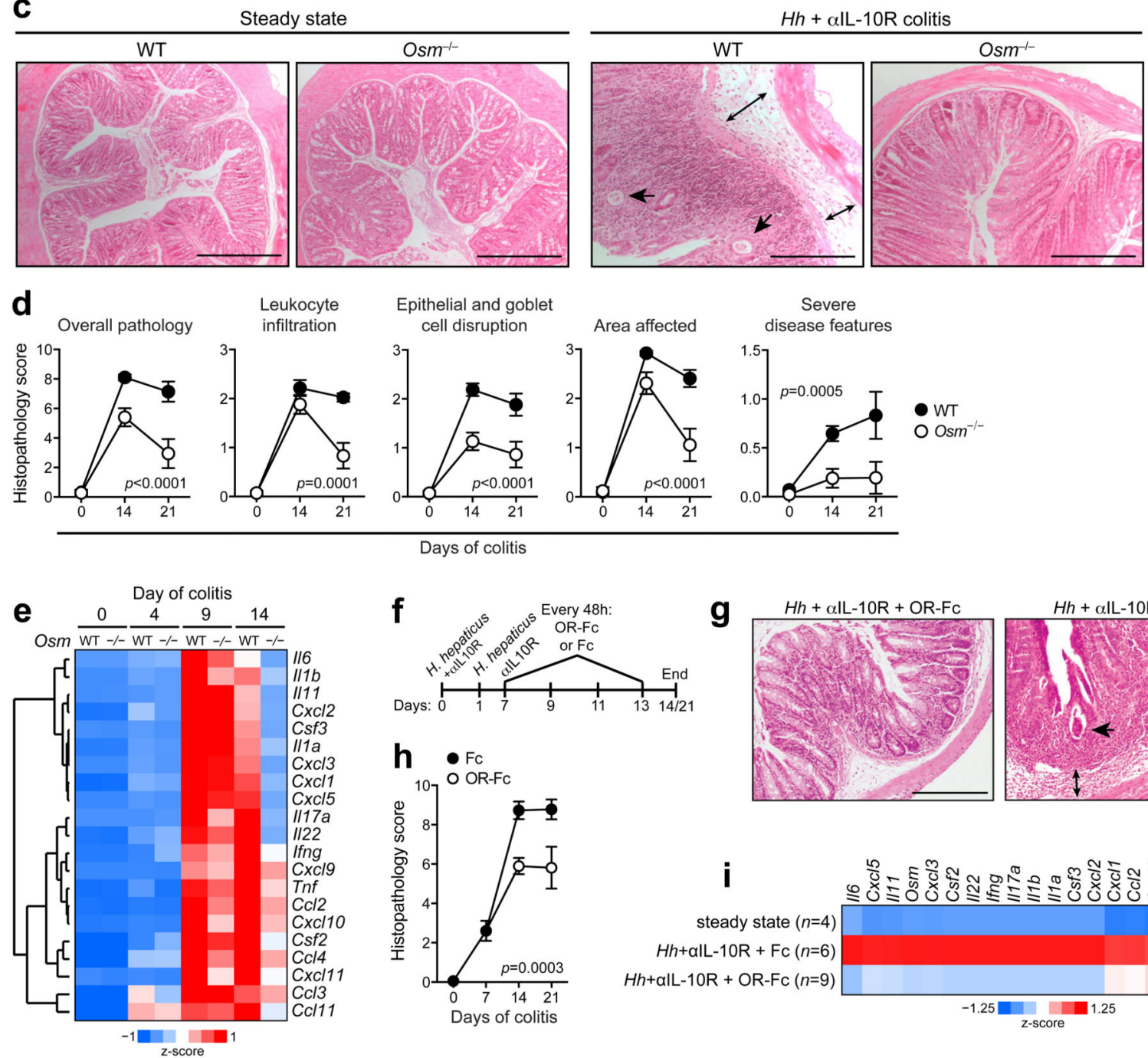

$\mathrm{O} \mathrm{Osm} \mathrm{sm}^{-1-}$

Figure 6. OSM promotes anti-TNF resistant colitis in vivo.

(a-d) $H h+$ aIL-10R colitis in wild type C57BL/6 mice and $\mathrm{Osm}^{-1-}$ littermates. (a)

Representative colonoscopy images at day 21, with endoscopic pathology scores shown in panel b (compared using Mann-Whitney $U$-test). Data represent one of three independent experiments. (c) Representative H\&E stained mid-colon cross-sections of healthy mice and colitic animals (day 21). Single arrows, crypt abscesses; double-arrows, submucosal edema.

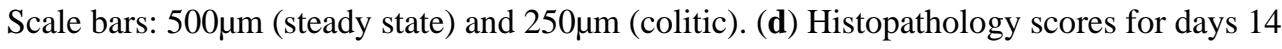
and 21. $n \geq 7$ mice per time-point, pooled from three experiments. $P$-values reflect differences 
between genotypes and are derived from two-way ANOVA. (e) Cytokine and chemokine mRNA expression in whole colon tissue from mice subjected to $H h+a$ IL-10R colitis for 4, 9, or 14 days ( $n=4-6$ per group). Expression was averaged for mice within each genotype and timepoint group, and converted to z-scores. (f) Therapeutic blockade of OSM in the $H h$ $+a \mathrm{IL}-10 \mathrm{R}$ model. OR-Fc (150 $\mu \mathrm{g}$ every two days) or a molar-equivalent dose of Fc control protein were injected intraperitoneally starting at day 7. (g, h) Representative H\&E stained mid-colon cross-sections of OR-Fc or Fc treated mice and associated histopathology scores ( $n=5-13$ mice per group, pooled from three experiments). $P$-values reflect differences between treatments and are derived from two-way ANOVA. Single arrows, crypt abscesses; double-arrows, submucosal edema and inflammatory infiltrate. Scale bar=250 $\mu \mathrm{m}$. (i) Expression of the OSM-associated inflammatory module in colons of mice treated as depicted in panel $\mathbf{f}$. Data represent one of three independent experiments. 